Title:

\title{
Sensing Local Field Potentials with a Directional and Scalable Depth Array: The DISC electrode
}

Authors:

Amada M. Abrego ${ }^{1} \dagger$, Wasif Khan ${ }^{1} \dagger$, Christopher E. Wright ${ }^{1,2}$, Neela Prajapati ${ }^{1}$, M. Rabiul Islam ${ }^{1}$, Mohammad H. Ghajar ${ }^{1}$, Xiaokang Bai ${ }^{1}$, Nitin Tandon ${ }^{1}$, John P. Seymour ${ }^{1,3 *}$

Affiliations:

${ }^{1}$ Department of Neurosurgery, University of Texas Health Science Center, Houston, TX, 77030 USA

${ }^{2}$ Department of Bioengineering, Rice University, Houston, TX, 77030, USA

${ }^{3}$ Department of Electrical and Computer Engineering, Rice University, Houston, TX, 77030, USA

† Co-first authors

${ }^{*}$ Correspondence: John Seymour (john.p.seymour@uth.tmc.edu)

\section{Abstract}

A variety of electrophysiology tools are available to the neurosurgeon for diagnosis, functional therapy, and neural prosthetics. However, no tool can currently address these three critical needs: (i) access to all cortical regions in a minimally invasive manner; (ii) recordings with microscale, mesoscale, and macroscale resolutions simultaneously; and (iii) access to spatially distant multiple brain regions that constitute distributed cognitive networks. We present a novel device for recording local field potentials (LFPs) with the form factor of a stereoelectroencephalograhic electrode but combined with radially positioned microelectrodes and using the lead body to shield LFP sources, enabling directional sensitivity and scalability, referred to as the DISC array. As predicted by our electro-quasistatic models, DISC demonstrated significantly improved signal-to-noise, directional sensitivity, and decoding accuracy from rat barrel cortex recordings during whisker stimulation. Critically, DISC demonstrated equivalent fidelity to conventional electrodes at the macroscale and uniquely, revealed stereoscopic information about current source density. Directional sensitivity of LFPs may significantly improve brain-computer interfaces and many diagnostic procedures, including epilepsy foci detection and deep brain targeting. 


\section{Introduction}

For both clinical research and systems neuroscience, the question of recording scale in time and space is a fundamental and ongoing challenge. What spatial resolution is required to elucidate the etiology or diagnosis of this disease? Or what scale will best help us understand the neurobiology of a given neural system? The enthusiasm for more data, is balanced by practical questions of safety and current limits of technology. Non-invasive field potentials (electroencephalograms (EEG) and magnetoencephalograms (MEG)) are used to measure the health or dysfunction of electrical activity within the brain. When high spatial precision is required, subdural surface electrodes (a field potential known as electrocorticogram (ECoG)) and/or depth electrodes are used instead of EEG/MEG (Kovac et al. 2017). The commonest intracranial recording scenario in humans occurs in patients with drug-resistant epilepsy with focal seizures. These procedures were once based on surface arrays (i.e., ECoG). Beginning in France in 1965 (Bancaud et al. 1965), slowly spreading in Europe, and finally being adopted in the U.S. in the last decade, ECoG recordings have generally been replaced with stereoelectroencephalogram (sEEG) (Engel 2018) for most diagnostic surgeries. sEEG is a specific form of depth electrodes typically with eight to sixteen ring electrodes. Given modern pre-op imaging tools and frameless robotic guidance, its advantages over the ECoG grid are reduced risks of hemorrhages, infection, and headaches (Tandon, Tong, Friedman, Johnson, Von Allmen, Thomas, Hope, Kalamangalam, Slater, and SA 2019; Yan et al. 2019; Sacino et al. 2019; Toth et al. 2019; Yang et al. 2017; Jehi et al. 2021). Furthermore, there is less skull damage during the procedure, and significantly greater resolution in the measurement of signals not originating near the surface. These advantages have been impactful, for example, in the diagnosis of medial temporal lobe epilepsy. Resective surgery in one sEEG cohort resulted in an absolute $21 \%$ greater seizure freedom relative to ECoG grids (Tandon, Tong, Friedman, Johnson, Von Allmen, Thomas, Hope, Kalamangalam, Slater, and Thompson 2019). In short, stereotactically placed depth electrode arrays possess an excellent form factor for minimally invasive large-scale recording and stimulation, yet concurrently enable an interface with broadly distributed cortical networks. The success of depth arrays implies their use in more applications such as a brain-computer interface $(\mathrm{BCl})$ although the decoding performance of the sEEG (Herff, Krusienski, and Kubben 2020; Angrick et al. 2020) is still trailing that of surface grids 
(Makin, Moses, and Chang 2020; Moses et al. 2021; Hammer et al. 2016) and the Utah array (Willett et al., n.d.; Thomas et al. 2020; Hochberg et al. 2012)(Willet; Crone; Hochberg). As we will demonstrate, the resolution of sEEG electrodes in their current form is limited by the circumferential nature of the ring electrode, and a redesign of the form and scale of these electrodes would enable the creation of a platform technology to enable many applications. Besides field potentials, researchers have two other temporally precise recording domains: extracellular single cell $(300-5000 \mathrm{~Hz})$ and local field potentials $(<300 \mathrm{~Hz})$. The former is generally preferred in systems neuroscience and $\mathrm{BCl}$ but measure cell-driven currents measurable within 100-120 $\mu \mathrm{m}$ of the source (Du, Roukes, and Masmanidis 2009). This limits the volume and loci of cortex actually sampled by these electrodes. Local field potentials recorded using microelectrodes, provide a spatial resolution between the field potential and single cells. Local field potentials are an amalgam of distant sources coupled with adjacent cellular structures (Lindén et al. 2011), thus suffering from a "cocktail party" problem. Relative to spiking single cells, the many simultaneous sources, limited signal-to-noise ratio (SNR) and directional insensitivity make source separation challenging, though an active area of research (Oosugi et al. 2017; Lucka et al. 2012). So, for researchers and clinicians, the choice between extracellular recordings versus LFPs includes a tradeoff of signal fidelity and the volume of tissue measured.

Exactly what neuronal signal are measurable using FPs and LFPs is an ongoing question and a function of the electrode and device geometry, and the distance from the source (Pesaran et al. 2018; Lindén et al. 2011; Kajikawa and Schroeder 2015). The largest signals are driven by synaptic transmembrane currents in populations of neurons whose geometry most strongly approximates an open-field dipole (Nunez and Srinivasan 2006; Buzsáki, Anastassiou, and Koch 2012). Layer $V$ neurons throughout mammalian neocortex have been demonstrated in biophysical models to accurately account for microelectrode-based LFP recordings (Hagen et al. 2018; Dura-Bernal et al. 2019), and EEG and MEG signals (Neymotin et al. 2020; Næss et al. 2021). As predicted, the LFP from even a small, localized source in macaques can travel 6 $\mathrm{mm}$ before it is attenuated by $50 \%$ (Kajikawa and Schroeder 2011). Critically, the information recorded at a microwire does not contain directional information, although it could be triangulated if the spatial spread of an array of microwires and SNR was sufficient. Source separation, and the harder problem of localization, work best with spatially diverse electrodes. In fact, despite providing single unit data, microelectrodes are more susceptible than macroelectrodes to the cocktail party problem because, unlike large ring electrodes, these 
remain highly sensitive to the diverse, local, and laminar sources of the immediate cortical column (Herreras 2016). Conversely, a ring macro-electrode records the weighted sum of all voltage contributions across its surface (all 360 degrees) and this greatly dilutes immediately local inputs. To summarize, this limited source separation of LFPs limits its usefulness in neuroscience, neurology, and brain-computer interfaces (BCls), especially confounding our resolution in the mesoscale -0.1 to $5 \mathrm{~mm}$.

We describe a novel multi-scale recording array capable of stereo-LFP and current source density measurements. The directional and scalable array, or DISC, designed to overcome the limitations described above, provides much needed mesoscale source separation. We performed finite element modelling based on electro-quasistatic physics and an in vivo recording in the rat barrel cortex, which was chosen for spatially segregated cortical columns. We performed insertion through a small twist-drill hole similar to the sEEG procedure which takes only a few minutes per electrode in humans (Rollo et al. 2021). An under-appreciated phenomenon, "substrate shielding", was applied to spatially segregate LFPs. Others before us were the first to identify the phenomenon but on a smaller scale. Two reports (Anderson, Oweiss, and Bierer 2001; Moffitt and Mclntyre 2005), and later others and ourselves (Seymour et al. 2011) describe how neurons can be shielded by the device, resulting in directional sensitivity. Since that work, Boston Scientific has begun offering directional electrodes in the form of a ring with 3 segmented sections across a $1.2 \mathrm{~mm}$ diameter DBS lead body. Recently, detailed modeling by Noor and Mclntyre (Noor and Mcintyre 2021) describes the quantitative advantages of the segmented electrodes versus ring electrodes in providing directionality. This report expands these concepts in a more generalizable design and establishes why microelectrodes on larger substrates may be transformative to neuroscience and clinical diagnosis. We quantify the contributions of substrate diameter, electrode size, source orientation, and distance on signal isolation. In addition to high quality, multidirectional CSD, mesoscale source separation, DISC also provides ring-equivalent recordings. Finally, we describe several manufacturing approaches for making DISC in a way that is compatible with the goal of being concurrently useful as a standard of care stereotactic electrode. 


\section{Results}

\subsection{Electro-quasistatic model of substrate shielding and directional sensitivity}

Maxwell's equations can be reduced to an electro-quasistatic model with the current conservation law applied to an array of sinks and sources in the extracellular space (Hämäläinen et al. 1993; Baillet, Mosher, and Leahy 2001). These results apply to all frequencies of clinically relevant extracellular potentials. The Poisson equation,

$$
\boldsymbol{\nabla} \cdot \mathbf{J}=\boldsymbol{\nabla} \cdot(\sigma \boldsymbol{\nabla} \phi), \quad(\text { eq. } 1)
$$

where $\mathrm{J}$ is current density, $\sigma$ is conductivity, and $\phi$ represents the extracellular electric potential, can be applied to arbitrary current sources in a conducting volume with boundary conditions including the insulating body of a recording device.

\section{Directionality as a function of substrate diameter}

To compare microwire, ring electrode, and the directional and scalable (DISC) array, we modeled a dipole approximating a cortical column from layer $\mathrm{V}$ pyramidal cells (1.2-mm height and $1.67 \mathrm{~A}-\mathrm{m} / \mathrm{m}^{2}$ current density) (Murakami and Okada 2015) (Fig. 1). The FEM package ANSYS was used throughout this study to model device geometries and a variety of current source orientations and distances. A useful measure for the "directionality" of a source and device combination is the change in voltage between nearby electrodes. Directionality is conveyed as the front voltage to back voltage ratio $(F / B)$, which is dominated by substrate diameter. The normal declination of voltage should be between $1 / r$ and $1 / r^{2}$ depending on the geometry of the sources, but the F/B ratio is significantly higher than previously expected and grows nonlinearly with the diameter (Fig. 1C). Large diameter insulating bodies create a disturbance in the voltage contours unlike smaller single unit recording tools (e.g. Benke-Fried wires (Fried et al. 1999), IMEC probe (Jun et al. 2017) or Utah array). Since this demonstration creates an increasing distance between electrode pairs, it is important to compare this ratio when lead body conductivity matches the value of tissue ( $\sigma=0.26 \mathrm{~S} / \mathrm{m}$ ) (Fig. $1 \mathrm{C}$ green line). The difference between these lines represents the effect of "substrate shielding" on the dipole current, which is caused by diverting the current around the lead body (Suppl Fig. 1). The isopotential around the front electrode extends toward the source, and the isopotential around the back electrode extends away from the source because current is not flowing in the immediate vicinity. These combined effects provide a previously undescribed directionality 
available to a larger diameter device when recording field potentials from synchronous sources even at considerable distances.
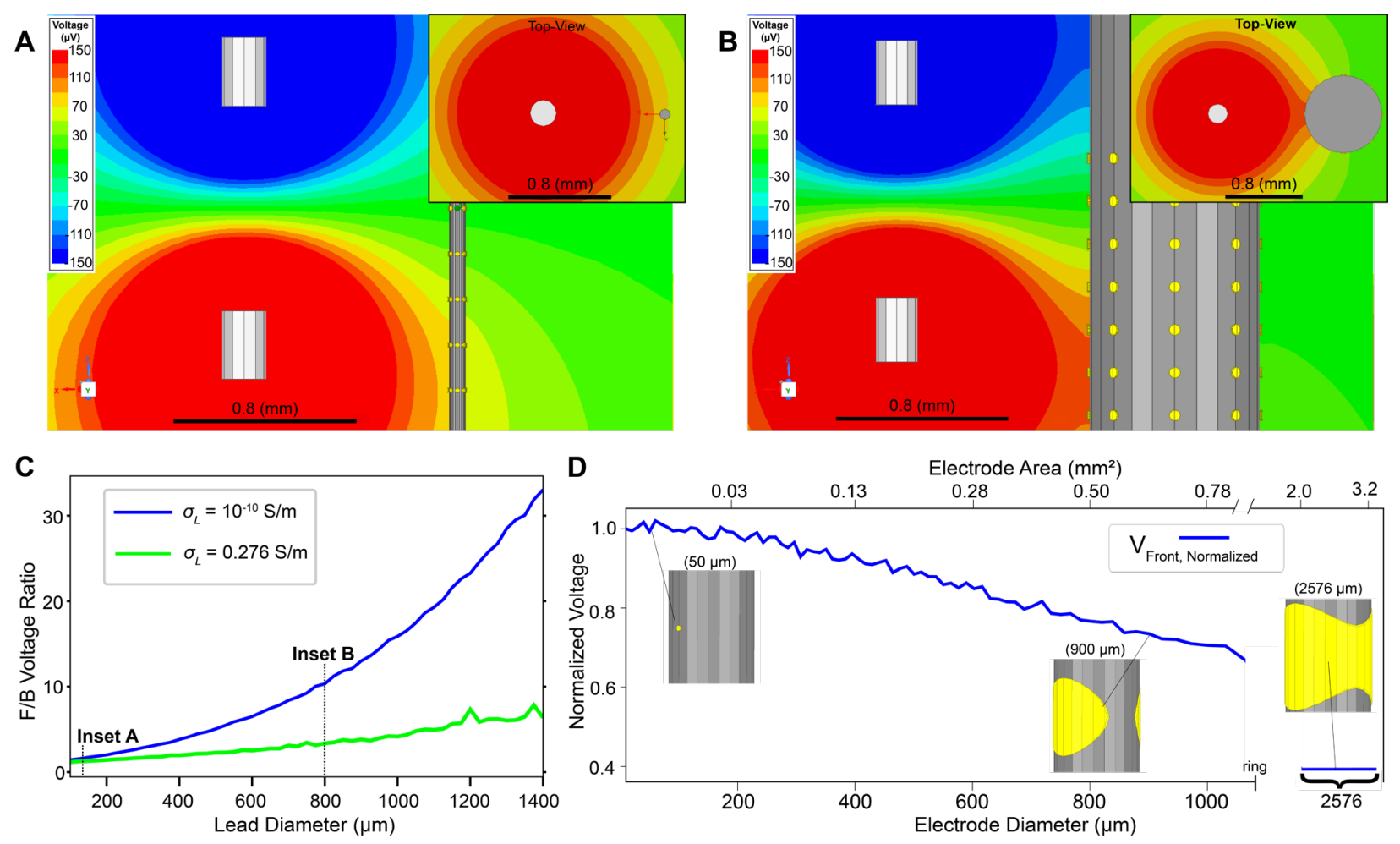

Figure 1. Directionality and amplitude as a function of substrate and electrode diameters.

A, A 65- $\mu \mathrm{m}$ diameter and B, 800- $\mu \mathrm{m}$ diameter substrate with independent electrodes on opposite sides at a fixed distance from a dipole source. Voltage $V$ from a current source shown to left of the lead body represents activity in layer V pyramidal cells in the rat cortex $(200-\mu \mathrm{m}$ diameter) generated in a finite element model (ANSYS). Voltage is inversely related to distance and perturbed by changes in conductivity $\sigma$ in space created by the insulating body. C, ANSYS model of the front to back electrode voltage ratio as a function of diameter $\left(\varnothing_{\mathrm{sh}}\right)$. When $\sigma$ of the lead body matches local tissue ( $\sigma=0.26 \mathrm{~S} / \mathrm{m}$ ), F/B increases due to the increasing distance between front and back electrodes. Substrate shielding magnifies the difference between the front and back electrodes. D, Normalized voltage amplitude as a function of electrode diameter. The source was identical as before. Attenuation becomes significant beyond $120 \mu \mathrm{m}$. At 1238 $\mu \mathrm{m}$ (clinical depth electrode e.g., sEEG dimensions), a ring forms and amplitude is attenuated by $60 \%$ relative to a microelectrode. Increasing the ring diameter any further has negligible attenuation. A video of this effect is provided in Suppl. Movie 1. 


\section{Electrode diameter and LFP source amplitude}

Large electrode surface areas are most practical in noisy clinical environments given their low impedance and therefore poor EMI pickup. We parameterized the electrode diameter on a 800$\mu \mathrm{m}$ lead body and measure the peak amplitude when a dipole and the nearest electrode have a gap of $0.8 \mathrm{~mm}$. The decline in amplitude with increasing diameter is quite significant even before the electrode wraps around the substrate to form a ring (Fig. 1D). Once the ring forms, the area no longer has any significant affect. A $120-\mu \mathrm{m}$ diameter microelectrode $\left(11,300 \mu \mathrm{m}^{2}\right)$ has only a theoretical loss of amplitude of $3 \%$ for dipole-equivalent LFP sources. A minor attenuation may be an excellent tradeoff given some degree of EMI and thermal noise immunity, and therefore achieve greater SNR. Clinically available directional leads (Vercise, Boston Scientific; Sensight, Medtronic) have a diameter of $1.2 \mathrm{~mm}$. This model predicts that even a 1$\mathrm{mm}$ diameter electrode will be attenuated by $27 \%$ relative to even a $120-\mu \mathrm{m}$ electrode. Given that the actual electrode is a $1.5-\mathrm{mm}$ tall rectangle, the attenuation is likely greater).

\section{Source geometry and orientation}

The model so far considered an orientation most likely encountered when the array implantation is normal to the neocortical surface and parallel to cortical columns. Many device trajectories (Vakharia et al. 2017; Rollo et al. 2021) and therefore dipole orientations are possible. Open field dipoles may also appear as local sinks or sources (monopoles) and represent the bestcase attenuation $(\sim 1 / \mathrm{r})$. Each of these situations can create a different directional sensitivity (i.e., F/B ratio) and attenuation profile. We analyzed several possibilities: a monopole (200 $\mu \mathrm{m})$, dipole $(200 \mu \mathrm{m})$, large dipole $(700 \mu \mathrm{m})$, and orthogonal dipole all at varying distances from 0.15 $\mathrm{mm}$ to $5 \mathrm{~mm}$, defined as the mesoscale, by Nunez et al. (Nunez, Nunez, and Srinivasan 2019). The F/B ratio over distance for each is a useful quantitative juxtaposition. As expected, a parallel-oriented dipole has the greatest directionality but the least range ( $3 \mathrm{~dB}$ distance equals $1.9 \mathrm{~mm}$ ). The monopole and orthogonal dipole performed similarly with a $3 \mathrm{~dB}$ distance equal to $4.0 \mathrm{~mm}$ ) (Suppl. Fig. 2, Suppl. Movie 2).

\section{Directionality in a noisy multi-source environment}

In another model, we introduced eight cortical sources within $\sim 5 \mathrm{~mm}$ of the devices (Fig. 2), added gaussian noise at each electrode, and measured the SNR during an evoked potential 
task to isolate each source based on phase (see Methods). The amplification of the voltage from a dipole with its nearest electrode is direction dependent, meaning any source from a significantly different phase angle is attenuated by some degree thereby reducing the biological noise. Our simulation provides a challenging scenario by varying distance and diameter dramatically (Fig. 2A, B, Suppl. Table 2). Source 1 for example has two other sources within 20 degrees and therefore communicating over the same column of electrodes. Similarly, source 3 and 4 , and 5 and 6 have similar angles. Source 1 at $1 \mathrm{~mm}$ has an SNR $=1.8 \mathrm{~dB}$ with no repeated trials and $7.6 \mathrm{~dB}$ after 50 trials (Fig. 2C). For an ultra-high-resolution ring electrode (0.4-mm tall, 0.8-mm diameter) the SNR was 0.38 and $3.9 \mathrm{dBV}$. Note that 2-mm tall ring electrodes are standard, and these would have performed considerably worse given the narrow peak of voltage from a dipole (Fig. 1). This difference exists despite the addition of a $4.3 \mu \mathrm{Vrms}$ (root mean square) on DISC microelectrodes, whereas the ring electrode included only 2.7 $\mu \mathrm{Vrms}$. DISC does not always have lower noise (data not shown) but does have greater SNR for each of the 8 sources. The most distant sources, except the large source 7 , had relatively modest improvement for DISC in this source configuration. CAR improves SNR for the farthest sources by subtracting out biological background noise. The ring electrode outperforms a microwire for all but the closest source because of its lower noise floor. A 2-mm tall ring electrode was added for completeness and its large size reduced the amplitude for all but two sources (Suppl. Fig. 3). Arrays of microelectrodes would have also benefited from CAR (Ludwig et al. 2009) although not tested here. An additional model of tissue encapsulation conductivity and thickness was performed (Suppl. Fig. 4). Encapsulation tissue improved the recording amplitude slightly as predicted in Moffitt, Mclntryre 2005 (Moffitt and Mclntyre 2005).

Virtual electrode geometries can be computed with digital averaging and referencing (Blanche et al. 2005). Generating an equivalent macroelectrode signal is important because of its long clinical history and effective filtering in certain frequencies (Nunez and Srinivasan 2010). We validate this ability theoretically using ANSYS and the previously described multi-source and noise simulation model. For each of the 8 sources in our model, we measured both the ring and virtual ring (comprised of $3 \times 8$ electrodes at the same depth) and found DISC performed nearly equivalently across sources (Suppl. Fig. 5). 

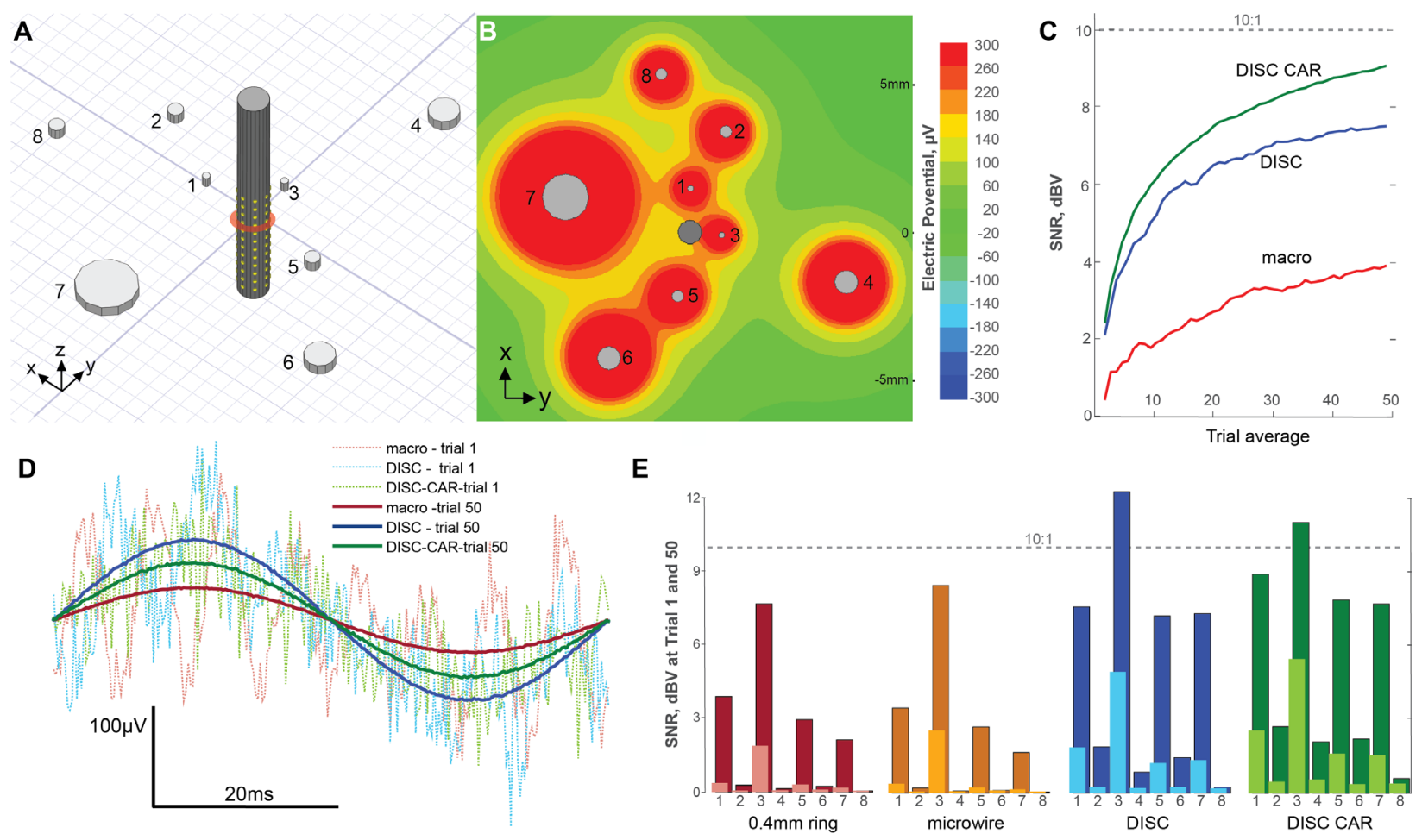

Figure 2. Electro-quasistatic 3D dipole model demonstrating directional sensitivity in a multi-source configuration. A, 8 simultaneous dipoles modeled in FEM with identical surface boundary current density (only sink is shown, $0.5-\mathrm{mm}$ grid). Three device types are modeledDISC (shown), microwire, and 0.4-mm tall ring electrode. B, Voltage heat map through layer $\mathrm{V}$ dipoles with sources on at peak current $\left(1.67 \mathrm{nA}-\mathrm{m} / \mathrm{mm}^{2}\right)$. Heat map is at the plane intersecting the dipole sinks. C, Signal-to-noise (dBV) for macro, DISC, and DISC with CAR during trial 1 and cumulative trials. D, Waveform examples for 1 and 50 trials for the macro and DISC electrode when phase locked to Source 1. Sources 2-8 are assigned a random phase and frequency. Assigned noise of $2.7 \mu \mathrm{Vrms}$ and $4.3 \mu \mathrm{V}$ to each ring or microelectrode, respectively. $E$, SNR comparison of the simulated potentials for each source independently phase-locked. Microwire $\varnothing=65 \mu \mathrm{m}$. Trial $1=$ light color; Trial $50=$ dark color (avg). 


\subsection{In vivo amplitude, SNR, and directional sensitivity}

\section{Amplitude and SNR}

One tetrode (50- $\mu \mathrm{m}$ bundle diameter) was implanted into 7 rats each with 9 whiskers being stimulated over 450 trials. Immediately following tetrode recordings, a DISC electrode with 128channels of $80-\mu \mathrm{m}$ electrodes wrapped on a rigid cylinder (650-720- $\mu \mathrm{m}$ diameter) was implanted $3.2 \mathrm{~mm}$ until the top electrode row was no longer visible (details in Methods) via the same $0.9-\mathrm{mm}$ twist drill hole using an identical trajectory (typically $38^{\circ}$ relative to the sagittal plane). We compared recordings from the tetrode, DISC, and two virtual ring electrodes created from DISC recordings. One ring type was a 0.4-mm tall $\times 3$ array and the other a 2-mm tall single electrode (mirroring the commercial standard). The 0.4-mm rings spanned infragranular, granular, and supragranular layers. The mean amplitude of the LFP signal of our DISC device $(250.6 \pm 182.5 \mu \mathrm{V})$ was significantly better than the tetrode $(86.6 \pm 65.5 \mu \mathrm{V}$, Tukey's test $\mathrm{p}<0.001)$, virtual $0.4-\mathrm{mm}$ ring $(120.2 \pm 97.6 \mu \mathrm{V}, \mathrm{p}<0.001)$, and the virtual $2-\mathrm{mm}$ ring $(59.1 \pm 63.3$ $\mu \mathrm{V}, \mathrm{p}<0.001$ ). Similarly, the DISC device significantly improved the LFP SNR (dBV) (DISC: $13 \pm$ 3.3 , tetrode: $7.9 \pm 3.3$, virtual $0.4-\mathrm{mm}$ ring: $10.3 \pm 3.5$, virtual $2-\mathrm{mm}$ ring: $7.3 \pm 4.0, \mathrm{p}<0.001$ ) (Fig. 3). The mean rms noise floor across subjects was $4.2 \pm 2.1$ and $5.5 \pm 3.9 \mu \mathrm{Vrms}(100 \mathrm{~ms}$ window prior to stimulus) for DISC and tetrodes, respectively. In practice, CAR improved the SNR (dBV) in trial averages, increasing it by 2 to $15.3 \pm 2.7 \mathrm{dBV}$ (Tukey's test, $p=0.001$ ). A lower amplitude was also noted for CAR (Suppl. Fig. 6), as expected from theory. All experiments were recorded in a Faraday cage. To illustrate noise immunity with CAR, we also recorded a session with an intentional ground loop and no Faraday cage, which in this example made a significant, observable difference (Suppl. Fig. 7). 

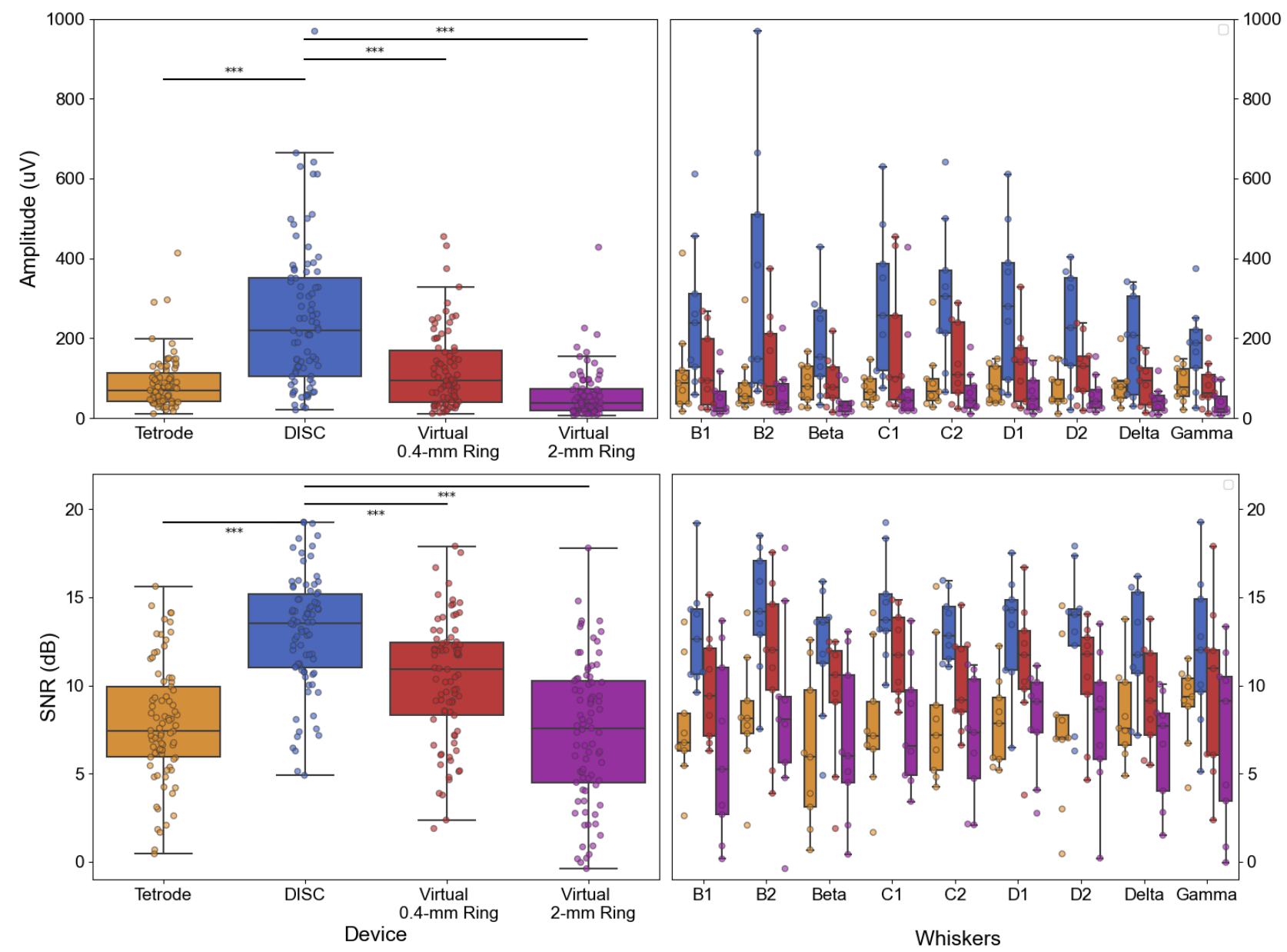

Figure 3. Amplitude and SNR for each device type. Ring electrodes were created virtually by averaging over a virtual ring of height $0.4 \mathrm{~mm}$ or $2 \mathrm{~mm}$. Vertical pitch of DISC array was 200 $\mu \mathrm{m}$. Whisker by whisker values are shown to the right. $\mathrm{N}=9$ subjects and 9 whiskers each.

\section{Directional Sensitivity and CSD}

Cytochrome staining was performed in some subjects to identify the device location. Histology from subject S6 indicated DISC and the microwire were implanted in barrel E1 (medial edge of barrel field). A directional voltage profile (detailed in Methods) for nine whisker stimuli also correlated to the position identified in histology (Fig. 4, Suppl. Fig. 6). We also modeled dipoles at the specific histological coordinates and diameter for each of nine barrels and plot the FEM predicted profiles (Fig. 4C). Our model assumed a perfectly confined excitation pattern instead of the expected lateral excitation between barrels (Petersen 2019; Peron et al. 2015), which would at least partially explain the broad profiles found in vivo relative to FEM. Directional 
profiles showed greater variance from trial to trial, as expected given independent electrode noise. Examples of the resultant vector measurement are provided for 3 subjects (Suppl. Fig. 9). A total of 3 rats with histological evidence was collected in this study and the placement and direction profile shows high consistency (Suppl. Fig. 10).

Results for local circuit measurements are still important especially in systems neuroscience. In several subjects with a known barrel location, we performed CSD analysis to evaluate its ability to conduct stereo-CSD measurements. CSD is a solution to equation [1] given known potential values across the lamina (Buzsáki, Anastassiou, and Koch 2012). The results demonstrate CSD from two opposing direction in an example using barrels D1 and gamma (Fig. 4D).
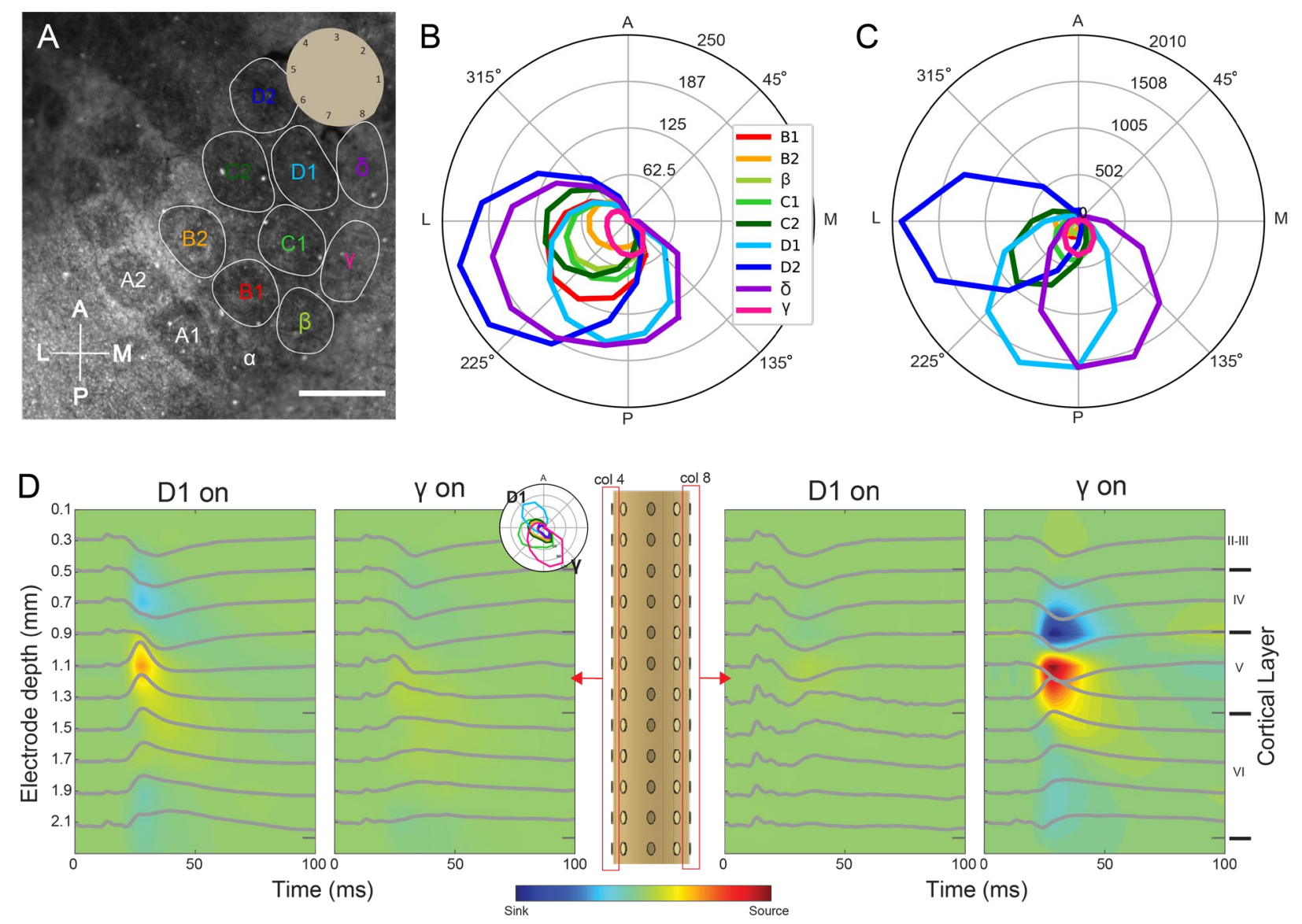

Figure 4. Demonstrating the directional sensitivity in rat barrel cortex. A, Example histology results using subject $\mathrm{S} 6$ and electrode layout from device 3. Unedited histology in Suppl. Fig. 8. Scale $=500 \mu \mathrm{m}$. B, LFP data from subject S6 and nine whiskers (depth=1.2mm). 
Directional polar plot formed from 8 channels, interpolated to 16 channels $\left(\sim 22.5^{\circ}\right)$, and subtracted the minimum amplitude. C, FEM results assuming nine dipole sources spaced in an identical location as shown in the cytochrome-stained image. D, Multi-directional CSD from DISC demonstrated in subject S7 when two independent primary whiskers are stimulated. Barrel D1 was located closest to device column 4 while barrel y was located on column 8 $\left(\sim 180^{\circ}\right)$. Polar plot of all whiskers shown in inset. Distinct CSD amplitude attenuation is observed. Average from 450 trials.

\subsection{Whisker decoding and information capacity}

Using the whisker stimulation experiment described above, we tested the accuracy of each device type in a 9-class discrimination task (Fig. 5). Our basic feature matrix prior to principal component analysis (PCA) consisted of the gamma band waveform (0:0.5:100ms) for each included electrode. Each row represented one trial. PCA was applied for dimensionality reduction before classification. Linear discriminate analysis and 10-fold cross-validation was performed for all classifications.

DISC accuracy when using 11 rows and 4 columns was an average of $93.6 \pm 7.7 \%$ and was significantly better than the tetrode, 0.4-mm x 3 ring array, and the 2-mm ring (Fig. 6, Suppl. Table 1). The 3-ring virtual device performed second best, and was significantly better than one $2 \mathrm{~mm}$ ring, which is the size of a standard sEEG. Each subject was analyzed independently where subject ID was a random effect in our generalized linear mixed model.

The classification accuracy of different electrode configurations and devices were tested and summarized in Suppl. Table 1. The best electrode configuration were 11 rows $x 8$ columns and 16 rows $\times 8$ columns, but the $11 \times 4$ configuration was not significantly different and thus used in the device comparisons (Fig. 6). Overall, the electrode configuration provided the strongest influence on DISC accuracy. Classifying with more than 11x8 channels has not yet been attempted. The 11 rows selected were in layers I to $\mathrm{VI}$ and the 4 columns were alternating to reduce redundancy. 
An important contrast is the DISC $1 \times 4$ configuration with the tetrode to separate out the effect of channel count. We chose 4 orthogonal electrodes on DISC in the same layer as the implanted tetrodes (1.4 mm deep). DISC significantly outperformed the tetrode with an accuracy of $71.2 \mathrm{vs}$ $58 \%(p<0.001)$. The amplification and substrate shielding affects can best explain this performance (Fig. 3).
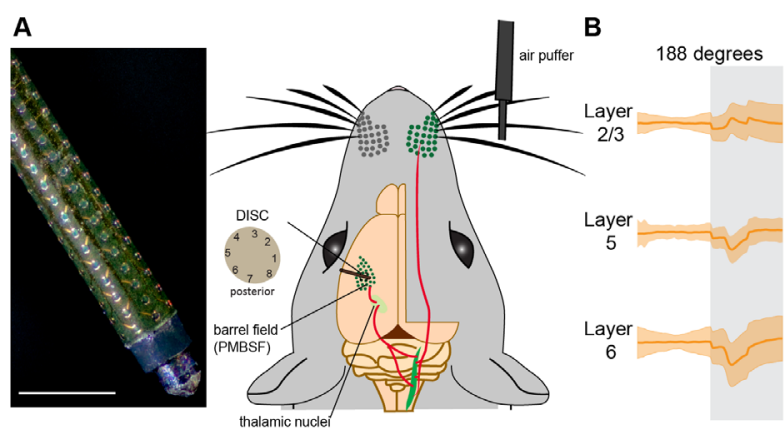

E

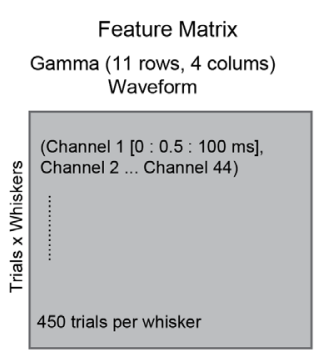

$\mathbf{F}$

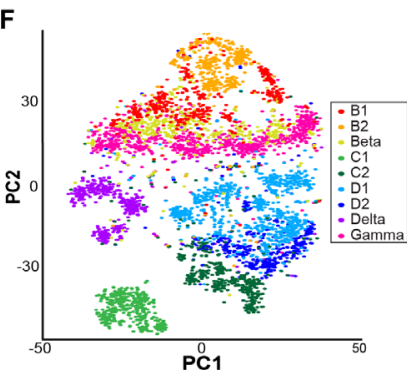

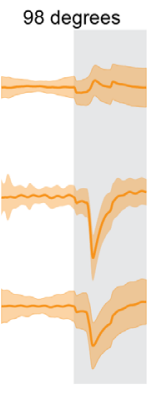

$\mathbf{G}$
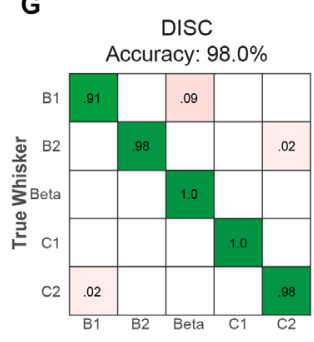

C
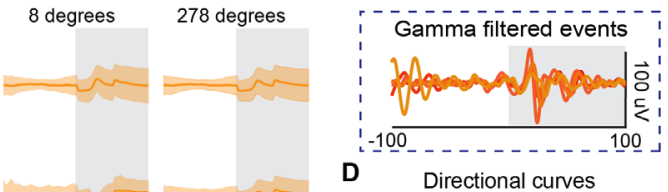

D Directional curves

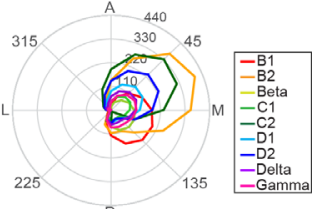

Virtual $0.4 \mathrm{~mm}$ macro Accuracy: $70.9 \%$

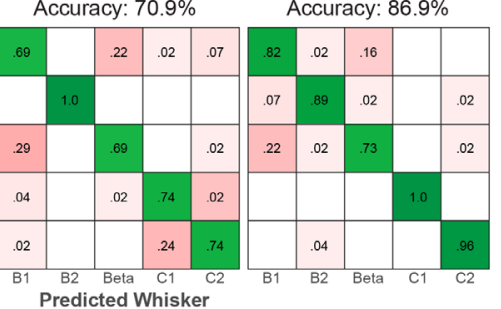

Figure 5. Methods and feature engineering overview for whisker stimulus discrimination in subject S4. A, Experimental design and DISC image. Scale $=1 \mathrm{~mm}$. B, Example 3x4 arrangement demonstrating evoked response across four directions in whisker B2. C, Example of gamma filtered response of B2 at 98 degrees. Individual trials represented with different colors. D, Polar plot. Directional curve calculated using the evoked response across eight directions for each whisker. Minimum value has been subtracted. E, Feature matrix arrangement. F, Dimensionality reduction and source separation using PCA. G, Example confusion matrix for three device types after analysis with a linear discriminant analysis (LDA) model. 

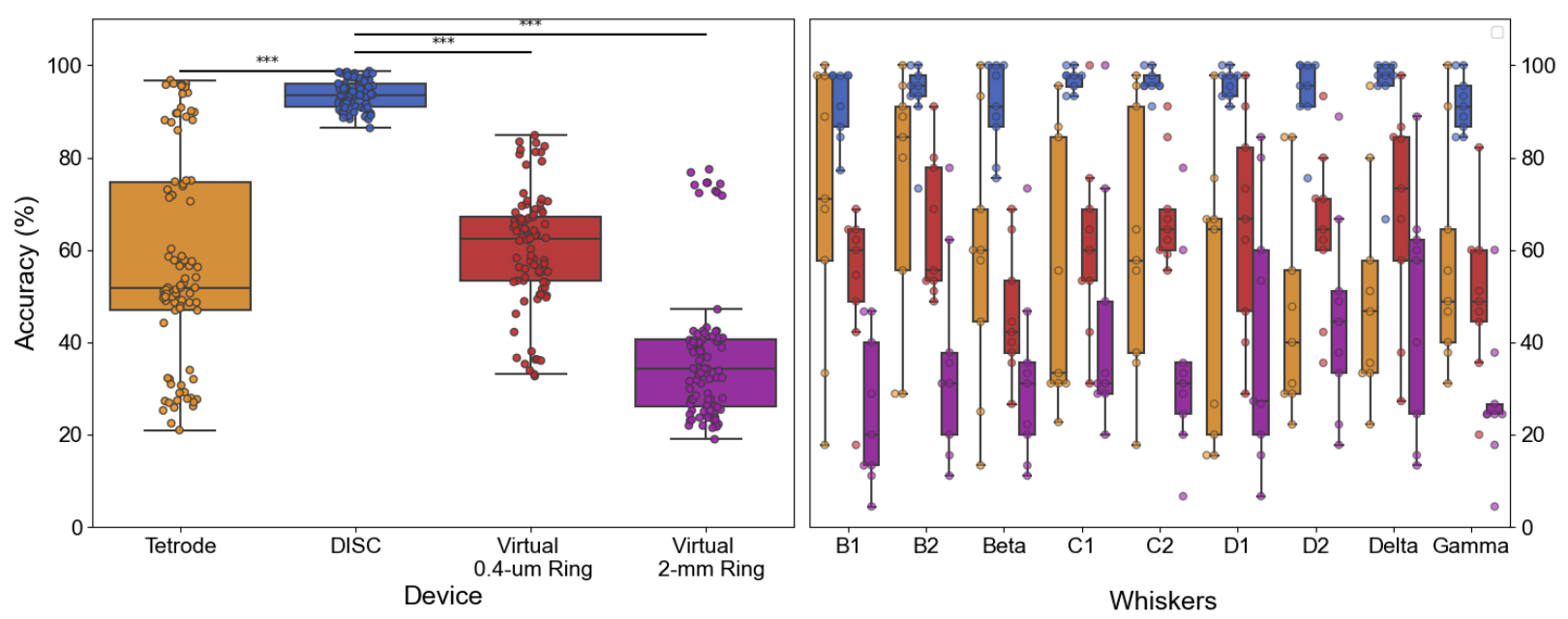

Figure 6. Decoding accuracy for each device type using a linear discriminant analysis model. ${ }^{* * *}$ denotes $p<0.001$, generalized mixed effects model, 9 subjects, 10 -fold crossvalidation). Whisker by whisker values are shown to the right,

\subsection{DISC manufacturing}

A variety of manufacturing methods were tested and conceived to improve the likelihood of translation. Prototype linear arrays were manufactured in our cleanroom at Rice University using a polyimide-metal-polyimide process as previously described ( $\mathrm{Na}$ et al.), but this resulted in poor axial alignment and complicates the backend design (Fig. 7A, backend not shown). Next we purchased similarly manufactured arrays from a commercial source originally designed for highdensity ECoG (Diagnostic Biochips, Inc) and used an assembly technique previously described (Pothof et al. 2016)(Fig. 7B). The first challenging aspect of this method is the tolerance in mold and device manufacturing makes fitting parts difficult. Also, if epoxy is used, the needed tight tolerance creates large capillary forces and often coats electrodes during the curing process. Another assembly approach is wrapping the array using heatshrink (details in Methods). Pattern a thin $(20 \mu \mathrm{m})$ adhesive sheet and mount on the backside of the 128-channel array. Prepare an insulated stainless-steel wire (432- $\mu \mathrm{m}$ diameter). Wrap the components using heat shrink and heat gun (Fig. 7C). This provided the greatest electrode yield and un-altered impedance values. There are many future options including wrapping on a silicone cylinder, direct molding of the PI array, and additive manufacturing. 


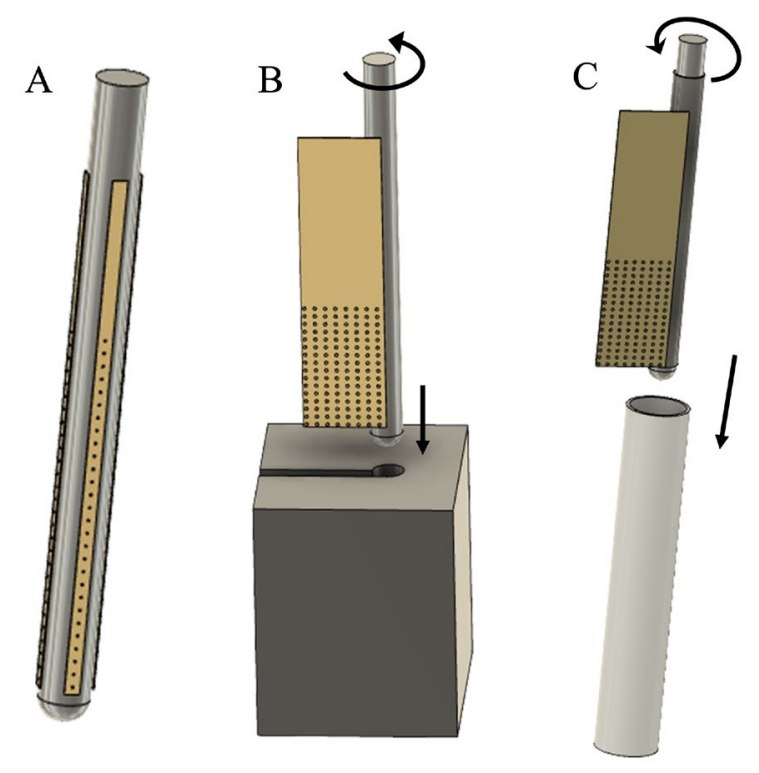

Figure 7. Example fabrication and assembly schematics available to practitioners. A, Linear polyimide arrays attached to rigid cylinder. B, Wrapping onto a rigid cylinder inside a rigid mold (originally proposed by (Pothof et al. 2016)). C, Wrapping method over a rigid body using heatshrink became our preferred method.

\section{Discussion}

Critically, DISC was effective at the whisker classification task at range despite its relatively narrow footprint. As predicted by the Shannon-Hartley theorem, the channels on DISC had higher SNR (Fig. 3) and thus the capacity to carry more information (Shannon 1948). This is supported by the virtual DISC-tetrode performance against the very small real tetrode. Also, in subject S6, the furthest barrel was $1.42 \mathrm{~mm}$ away $(\beta)$. The accuracy of this subject remained very high despite the barrel distance (92-100\%, 10-fold cross-validation, Suppl. Fig. 8). The spatial diversity of DISC provides a unique advantage relative to the other tested devices and yet it did not require a large craniotomy as would be necessary for a Utah array or an ECoG array. Like DISC, the virtual 0.4-mm ring array had unique layer information that likely contributed to its high accuracy. Unlike the other device types, DISC has directional information. The vertical span and the directionality are the most likely factors enabling decoding success. Having more electrodes, despite their redundancy, also proved to contribute significantly (Suppl. Table 1). This redundancy may have effectively denoised some part of the trial-by-trial noise. No 
trials were removed in any analysis. In an effort to identify the simplest and most discriminating features, 6 single statistics per trial and by channel were selected and used for classification. Of these, the root mean square of the evoked response provided the most accuracy for either DISC or tetrode (Suppl. Table 4).

Our results demonstrated for the first time, as far as we are aware, multi-directional CSD recordings. This capability may, for example, contribute to the understanding of lateral excitation or inhibition between cortical columns (Kato, Asinof, and Isaacson 2017). Encapsulation tissue may attenuate CSD results in chronic applications, which should be investigated. While many in the field cite a causal relation between encapsulation thickness and signal attenuation, the only published model on this topic (Moffitt 2005) predicts a complicated relationship even at the microscale. The local cortical columns will likely be damaged, but in a recent histological study around an $800-\mu \mathrm{m}$ sEEG ( $\mathrm{N}=3$ devices), the measured radius of tissue damage in a non-human primate was only $50 \mu \mathrm{m}$ for neuron density and $100 \mu \mathrm{m}$ for astrocyte reactivity (Gerbella et al. 2021). This is more damage than expected from most neuroscience tools such as a tetrode, silicon microelectrode, and especially fine, flexible single cell tools like the NET probe (Luan et al. 2017). For DISC the goal is to record LFPs which are naturally more stable in chronic applications than single units given their location (Perge et al. 2014; Flint et al. 2013). Only the most local LFP sources should be affected. Future work should conduct long-term recordings but also explore the parameters of resistivity and thickness inside of a more detailed biophysics/FEM model. We also do not suggest the use of DISC in mice given the lack of usefulness from other large devices such as a U-probe (300- $\mu \mathrm{m}$ diameter). The small twist-drill hole we use $(0.9 \mathrm{~mm})$ may be an important factor for its success in rats.

A particular advantage of DISC is its shape is similar to the stereo-EEG depth array, enabling its use in clinical environments with little alteration yet enabling more precise localization of seizures, and safety relative to grid arrays, as reviewed earlier. Thousands of epilepsy patients receive 10-20 sEEG devices each year for typically 1-2 weeks (Kaiboriboon et al. 2015). In one study reviewing 150 procedures, the robotic sEEG surgery averaged 81 minutes and 6.2 minutes per electrode (Rollo et al. 2021). The average surgery in this review implanted 13 sEEGs, having an insertion angle of $0-75^{\circ}$ with no significant change in registration accuracy based on angle. By contrast, the Utah array has only been implanted in approximately 21 persons with 1-6 implants each (Bullard et al. 2020). A single Utah array requires approximately 30-70-mm diameter skull opening for insertion. The cumulative neurosurgical experience and exceptionally low infection and hemorrhage rate when using sEEGs offer a safety advantage to 
non-stereotactic approaches (Herff, Krusienski, and Kubben 2020). We provide a summary of recent studies and meta-analyses in Suppl. Table 5, which highlights the safety advantages relative to ECoG.

The U-probe (Ulbert et al. 2001) can be viewed as a smaller version of DISC with a one-sided column of microelectrodes. The effect of substrate shielding is still measurable even at 300um (Fig. 1C). Our models of substrate shielding predicts that this lead body will attenuate many biological sources while amplifying those in the direction of the single column of microelectrodes. This laminar arrangement is ideal for CSD and its stereotactic insertion is several reasons for its usefulness in non-human primates. DISC provides these benefits but at a greater scale and multiple directions (Fig. 4).

Given we did not have access to a silicone cylinder of the right diameter, we used a stainless steel (SS316) core. A silicone- or polyurethane-molded cylinder would provide greater flexibility and that may result in greater signal stability in long-term applications (Wellman et al. 2017). Future work will include advanced manufacturing methods to improve flexibility.

\section{Conclusions}

This work demonstrates a highly effective, compact tool for improving amplitude, SNR, and decoding accuracy in a whisker decoding task. The decoding performance was due in part to the directional sensitivity that generated greater diversity of signals across the narrow array. The in vivo demonstration agreed well with the predictions of the electro-quasistatic model, despite the potential for local damage of DISC relative to a much smaller tetrode. These results also demonstrated accurate source separation and to a smaller degree, source localization (Fig. 4). More research is needed to demonstrate the source localization accuracy, especially for sources beyond a few millimeters. Two or more DISC arrays in tissue may be highly effective in future work and would benefit from finite element software like Brainstorm (Tadel et al. 2011). We will continue to improve the manufacturability of DISC and demonstrate its diagnostic capability in epilepsy models and advanced decoding tasks. We hope this work encourages the field to accelerate the trend toward smaller ring electrodes, directional leads, and ultimately toward high-density circular (DISC-like) arrays to explore the impact of LFPs "in stereo" and multi-scale. 


\section{Methods}

1. Modeling and simulation

ANSYS Electronics Desktop $2021 \mathrm{R} 1$ with the DC Conduction module was used throughout this work. A tissue block was modeled as $14 \times 14 \times 14 \mathrm{~mm}^{3}, \sigma_{\text {tissue }}=0.26 \mathrm{~S} / \mathrm{m}, \varepsilon_{\text {tissue }}=80$ (Koessler et al. 2017). Lead substrate was $6-\mathrm{mm}$ tall and a variable diameter, $\varnothing_{\mathrm{sh}}$, between 10 and 1400 microns. DISC diameter was by default $0.8 \mathrm{~mm}$ to emulate a standard sEEG device. $\sigma_{\text {shaft }}=1 \mathrm{e}-$ $10 \mathrm{~S} / \mathrm{m}$. $\varepsilon_{\text {shaft }}=2.7$. Cortical monopoles and dipoles had identical properties as tissue. A uniform current density of $I_{d}=1.67 \mathrm{nA}-\mathrm{m} / \mathrm{mm}^{2}$ was applied to the surface of all sources, which was derived from the work of Murakami and Okada demonstrating an invariant dipole current across animals of $Q=1-2 \mathrm{nAm} / \mathrm{mm}^{2}$, where $Q=I_{d} x d$ (Murakami and Okada 2015). We chose $Q=2$ $\mathrm{nAm} / \mathrm{mm}^{2}$ and a dipole distance, $d$, of 1.2-mm center to center. Unless otherwise stated, the dipole diameter was $200 \mu \mathrm{m}$. All microelectrodes and ring electrodes were platinum, $\sigma_{\mathrm{Pt}}=9.3 \mathrm{e} 6$ $\mathrm{S} / \mathrm{m}$. The ring electrode was $0.4 \mathrm{~mm}$ tall. Three contiguous surfaces of the tissue block outer surfaces were defined as $0 \mathrm{~V}$ to emulate a distant reference.

Figure 2 demonstrated 8 unique sources spread radially using an arrangement shown in Fig. 2A,B with details in Supplemental Table 2. That arrangement was intended to capture micro-, meso-, and macroscale sources interacting within a range of $5.1 \mathrm{~mm}$ in all directions. Sources 1 , 2 , and 8 , and separately 3 and 4 had overlapping angular positions and therefore used the same electrode column for best amplitude. For each trial, one primary source was assigned a phase, $\varphi=0 \pi$, and freq, $f=16.67 \mathrm{~Hz}$, while the other 7 sources a randomly assigned a random phase $(0 \pi$ to $2 \pi)$ and frequency $(40 \mathrm{~Hz}$ to $150 \mathrm{~Hz})$. Electronic noise is added to each device according to its electrode area and empirical data with electrodes of that size. Ring electrodes (0.4 mm tall) had a noise value of $2.7 \mu \mathrm{V}$ RMS. DISC had 96 electrodes each with an independent noise value of $4.3 \mu \mathrm{V}$ RMS. Both noise values were randomly generated using a Gaussian distribution. The waveform and SNR calculations were made as follows:

$$
\begin{aligned}
& \left.w_{e, \text { signal }}=\sum_{s=1}^{8} \operatorname{source}(t)_{s}+\text { noise }(t), \quad \text { (eq. } 2\right), \\
& \left.w_{e, n o i s e}=\sum_{S=2}^{8} \operatorname{source}(t)_{s}+\text { noise }(t), \quad \text { (eq. } 3\right), \\
& \left.S N R_{S}, d B V=10 \log \left[\frac{r m s\left(w_{e, \text { signal }}\right)}{r m s\left(w_{e, \text { noise }}\right)}\right], \quad \text { (eq. } 4\right),
\end{aligned}
$$


where index $e$ is specific to one electrode and device, and index $s$ is specific to a source.

Source amplitudes were sampled at the surface of the electrode. Source 1 in equations 2, 3 is, by definition, the primary or phase zero source. The primary source rotates for each of eight positions in Fig. 1A. SNR was computed for one cycle of the primary source.

The maximum amplitude of each source for each electrode and device type was computed by the ANSYS model as described before. The size and location of each source greatly influence the amplitude and noise contribution.

Suppl. Fig. 2 and Suppl. Movie 2 were a comparison of $\varnothing_{\mathrm{sh}}=70$ and $800 \mu \mathrm{m}$. Dipoles were either parallel or orthogonal and varied of a gap distance of 0.15 to $5 \mathrm{~mm}$. The "large dipole" source was $700-\mu \mathrm{m}$ diameter.

\section{Fabrication and assembly:}

a. Microwires (Tetrode)

Eight insulated 12 nichrome microwires (PX27, Sandvik AB, Sweden) were folded at a length of $8 \mathrm{~cm}$ to make a compact bundle approximately 50- $\mu \mathrm{m}$ diameter. During recording, the four highest SNR electrodes were chosen. One end of the bundle was mounted and taped on an extended clamp arm, and a small weight was applied. While the taped end of the bundle was kept static, the other end was coiled vertically using a tetrode spinner (Neuralynx) for 35-40 turns.

A thin layer of medical grade epoxy (Epoxy 301, Epoxy Technology) was applied on the wire bundle to provide additional strength and rigidity towards implantation. Heat $\left(250^{\circ} \mathrm{F}\right)$ was applied using a heat gun to cure the epoxy for 10 minutes. Tension was released from the coiled end and the bundle was allowed to untwist naturally. Post baking, both ends were cut with sharp scissors/ scalpel blades and the wire leads from the uncoiled end was manually connected to an Electrode Interface Board (Neuralynx EIB-36). The electrodes were electroplated in a gold noncyanide solution (Neuralynx) within an ultrasonic bath using an AutoLab Electrochemical Workstation (Metrohm), until a nominal impedance ( 250 kOhms) was achieved. The plated electrodes were cleaned with DI water, IPA and then air dried. 


\section{b. 128-ch DISC assembly}

Planar 128-channel arrays (G-128, Diagnostic Biochips, Inc, Maryland, USA) made of polyimide (HD Microsystems PI2611) formed the electrode body. G-128 model has $200 \mu \mathrm{m}$ and $250 \mu \mathrm{m}$ vertical and horizontal pitch, respectively, and follows a 16x8 matrix (row x column) configuration. Each electrode was $80-\mu \mathrm{m}$ in diameter and was coated with PEDOT. The thickness of the polyimide substrate with electrodes was approximately $12 \mu \mathrm{m}$. The planar electrode array was wrapped onto a guidewire/stylet using several techniques (Fig. 7A-C) to create a cylindrical lead body. Stainless steel (SS316) straight wire (GWXX-0170, 0.017" diameter, Component Supply Company) was used. To eliminate the possibility of the guidewire interfering with the electric field, PEBAX heat shrink tube (P2-023-0035-BLK, Cobalt Polymers, CA, USA) was applied on the SS wire. A 20- $\mu$ m thick double coated acrylic tape (UTD-20B(W), Nitto, NJ, USA) was utilized to wrap the flexible electrode array on the PEBAX coated guidewire. A FusionPro 48 Laser machine (Epilog Laser, Golden, CO, USA) was employed to pattern the thin adhesive film to match the shape of the G-128 probe. The thin adhesive film was placed on a flat substrate and fixed each corner with tape. Then running under $60 \%$ of Speed, $6 \%$ of Power, and $70 \%$ of Frequency with 2 cycles, the Vector mode from Laser machine could release desired adhesive film area within 20 seconds. Using a second heat shrink tube (PBST2-040-40-004C, Component Supply Company, USA), the electrode array was pressed firmly with the guidewire for better adhesion and to maintain the proper geometry. The guidewire tip was carefully machined to create a smooth insertion end for implantation with minimal blood vessel damage. Pre-implantation impedance was found to be (235 $\pm 244 \mathrm{k} \Omega$ ), while post implantation impedance was $(432 \mathrm{k} \Omega \pm 1.84 \mathrm{M} \Omega)$.

\section{Animal surgical procedure:}

A total of 9 Sprague Dawley rats (250-450 gm) were used in this study. Rats were housed in pairs in a regular $12 \mathrm{~h} / 12 \mathrm{~h}$ light/dark cycle with ad libitum access to food and water. All experimental procedures included analgesics and anesthesia and were approved by the Institutional Animal Care and Use Committee at Rice University. Rats were induced with 3-4\% isoflurane (SomnoSuite, Kent Scientific) and all whiskers on the right facial pad were trimmed except B1, B2, C1, C2, D1, D2, E1, beta, delta, gamma, which were selected for stimulation. Subjects were mounted firmly with ear bars on a stereotactic frame (Harvard Apparatus). Lubricating eye gel was used to keep the animal eyes hydrated. Topical lidocaine gel was 
applied on the ear bars to reduce pain during mounting and subsequent processes. Meloxicam $(5 \mathrm{mg} / \mathrm{ml})$ with a dose of $2 \mathrm{mg} / \mathrm{kg}$ was injected SQ as an analgesic. Animal body heat was maintained with isothermal heating pads. Hair was trimmed, and post application of topical ethanol and betadine, a 2-3cm rostro caudal incision was made to expose the skull. Hydrogen peroxide was applied to clean excess tissue and periosteum, while electrocautery was applied to stop any unwanted bleeding. Anesthesia was maintained with $1-2 \%$ isoflurane during the surgery.

Using a micro burr (FST, CA, US), a $4 \times 4 \mathrm{~mm}^{2}$ area of the skull was thinned on the left hemisphere. Brain images through the cranial window were captured with a CMOS sensor camera (CS126MU, Thorlabs, NJ, USA) mounted on a microscope (AmScope, CA, USA) using a 540nm light source to add contrast to the subdural vessels. Major blood vessels were avoided and a 900um twist drill hole was made near our estimated coordinates relative to bregma (C1 at -3.06 AP, 4.92 ML or D1 at -2.72 AP, 4.65 ML). These positions were adjusted proportionally for the nominal B-L distance of $8.8 \mathrm{~mm}$. A stainless-steel bone screw (\#0-80, Grainger, IL, USA) attached with a 32 AWG copper wire served as a reference electrode implanted above the cerebellum. The cranial burr hole was isolated using a layer of dental acrylic and silicone adhesive (Kwik-sil; World Precision Instruments, FL, USA), the reference electrode was isolated using dental acrylic only.

\section{Electrophysiology recording protocol:}

The whiskers were stimulated using a pneumatic dispenser (Nordson EFD Performus X, RI, USA). Each whisker was deflected by attaching a custom nozzle head to a standard $5 \mathrm{~mL}$ syringe placed $2.5 \mathrm{~mm}$ from root of the whisker. The air puff (10 psi) from the nozzle head created a $10 \mathrm{~ms}$ duration mechanical deflection in dorsal to ventral direction at $3 \mathrm{~Hz}$ over a $6 \mathrm{~s}$ stimulus and 6 s non-stimulus window; with a total of 450 stimulations recorded for each corresponding whisker. Neural signals from rat somatosensory cortex were recorded using our implanted set of microwire and DISC electrodes. Microwires were implanted at a depth of $1.4 \mathrm{~mm}$ from the dura surface to collect large amplitude signals, while DISC implantation reached a depth of $3 \mathrm{~mm}$ allowing the microelectrode array to be spanned over all cortical layers. Wideband signals were amplified, digitized and recorded using a 256 channels Intan RHD interface board (Intan Technology, CA, USA) using a sampling rate of $20,000 \mathrm{~Hz}$. The whisker stimulation was driven by an Arduino which also read into digital port of the Intan 
recording system. The Arduino was operated on a lithium-ion battery source and recordings occurred in a faraday cage unless otherwise noted.

Neural signals were analyzed using offline custom Matlab scripts (Mathworks, MA, USA).

\section{Data processing:}

Raw neural signals were down sampled at $2 \mathrm{kHz}$, low-pass filtered $(120 \mathrm{~Hz}$, local field potential (LFP)), and band-pass filtered (40-150 Hz, Gamma band). To account for signal variability, LFP and Gamma signals were isolated for each trial. The signal amplitude was calculated by finding the distance between peak and valley poststimulation. The SNR was computed by dividing the root mean square (RMS) of the signal post-stimulation by RMS of the pre-stimulus baseline.

Directional curves (polar plots) are defined by the peak-to-trough LFP amplitude and electrode column. A unique column orientation was noted for each device we assembled relative to the plane of the PCB. The plane of the PCB was always implanted parallel to the coronal plane. This angular information was used in the generation of polar plots. We matched the electrode amplitude of the average waveform to its angle. If an electrode was missing, the cubic interpolation was performed. A 16-point cubic interpolation was made from 8 electrode columns assume equidistance between each column. Directional curves were standardized by subtracting the minimum amplitude.

Resultant vectors (RV) were calculated by the angle position of the electrode and the peak-topeak LFP amplitude. The 8 original electrode positions were interpolated as described before to create 16 positions. The vectors were summed to create the resultant vector (length and angle). A ring electrode was simulated by averaging the signal of the electrodes in the DISC array. The number of electrodes used for averaging was determined by the desired macroelectrode vertical length. For a $2 \mathrm{~mm}$ virtual macroelectrode, we used all the channels that were included in a 2 $\mathrm{mm}$ vertical span (11 rows) of the DISC array. For the $400 \mu \mathrm{m}$ virtual macroelectrode, we used 2 adjacent rows of 8 microelectrodes each. The $0.4-\mathrm{mm}$ rings were chosen to span supragranular, granular, and infragranular layers, assuming the granular layer included the transition to a negative evoked potential. The gap between each row was $400 \mu \mathrm{m}$. 
We extracted the gamma band waveform from 0 to $100 \mathrm{~ms}$ poststimulation for each trial. The trial waveform from 44 electrodes, corresponding to 11 rows and 4 columns, were concatenated into one matrix. This was defined as our feature matrix with columns as the gamma waveforms, and rows as individual trials. To reduce the dimensionality of our feature matrix, Principal Component Analysis (PCA) was performed, choosing the number of components necessary to represent the $99 \%$ variance of our data. The reduced feature matrix was then divided into $10-$ folds. Each fold used $80 \%$ for training and $20 \%$ for testing with a linear discriminant analysis (LDA) model.

By calculating the second derivative of the average LFP waveform, we computed the current source density (CSD, (Schroeder, Mehta, and Givre 1998)) of each DISC row. For optimal results, the $20,000 \mathrm{~Hz}$ sampling rate waveform was used.

\section{Statistical analysis}

Generalized Linear Mixed Model (GLMM) was used to study the differences of DISC device (intercept) compared to tetrode and virtual rings. The subjects were used as random effects, and SNR, amplitude, or model accuracy as dependent variable. DISC was the defined baseline in the GLMM. When comparing between any two groups besides DISC, Tukey's range test was employed.

\section{Perfusion and Histology:}

A subset of rats had histology performed to identify the barrel location. Rats were euthanized by applying $5 \%$ isoflurane as a primary method, followed by cervical dislocation and thoracotomy as a secondary method and confirmation, respectively. The subset prepared for histology were euthanized using $5 \%$ isoflurane and cervical dislocation, followed by transcardial perfusion of 2\% PFA.

The cortex was flattened by scooping out the thalamus and placing in between two glass slides $\sim 1.5 \mathrm{~mm}$ apart. The flattened brain was kept in $1 \times$ PBS for 6-8 hours at $4^{\circ} \mathrm{C}$. It was later transferred to a $2 \%$ PFA solution for 24 hours. Fixed flattened brain was washed with $0.1 \mathrm{M} 1 \mathrm{X}$ PBS and was sectioned using a vibratome (Leica VT 1200S, Germany) to create $50-80-\mu m$ 
slices. Sections were transferred to a culture plate culture plate and rinsed in HEPES buffer $(0.1 \mathrm{M} \mathrm{pH} 7.4)$ for 15 minutes. Cytochrome oxidase staining solution was prepared similar to [(Lauer et al. 2018)] and the sections were incubated in the solution at $37^{\circ} \mathrm{C}$. Stains were visible around $30-60$ mins. $2 \%$ PFA was added with the solution to stop the staining reaction. Sections were mounted on microscope glass slides and rinsed in consecutive $70 \%$ ( 2 mins), 96\% (2 mins) and 99.5\% (3 mins) ethanol. Sections were rinsed in isopropyl alcohol for 3 mins, followed by xylene rinse for 5 minutes to complete the dehydration process. Applying a non-aqueous mounting media, the sections were cover-slipped and imaged using a microscope (Keyence BZX800, Japan) and 20X objective. Electrode implantation location was reconstructed from the slides containing stained barrel cortex maps.

\section{Acknowledgements}

We would like to acknowledge John Mosher (UTHealth) for helpful discussions on source localization and dipole modeling. We thank the staff at Rice University's nanofabrication facility for supporting our neural probe development. We thank Charlie Schroeder for his insights into local field potential recording with varied devices. We acknowledge the Texas Institute of Restorative Neurotechnologies at UTHealth for its financial support.

\section{Supplementary Information}

Table of Contents

Suppl. Table 1. LDA classification accuracy by device and electrode configuration

Suppl. Table 2. Source configuration table for a multi-source model (see Fig. 2).

Supp. Table 3. Electrical properties of electrostatic model.

Suppl. Table 4. LDA classification accuracy (\%) with different types of individual statistics estimated from gamma-band, DISC and tetrode

Suppl. Table 5. Review of Recent Safety and Efficacy Differences in Epileptogenic Diagnosis using ECoG vs SEEG

Suppl. Fig. 1. 2D J-field and Voltage contour illustrating the mechanism of substrate shielding Suppl. Fig. 2. Front/back amplitude by source type and orientation (related to Suppl. Movie 2) 
Suppl. Fig 3. SNR results for an 8-source model with a 2-mm ring electrode.

Suppl. Fig. 4. SNR, amplitude, and noise comparison between a FEM ring and virtual DISC ring electrode.

Suppl. Fig. 5. ANSYS model of encapsulation around 800- $\mu \mathrm{m}$ diameter lead body

Suppl. Fig. 6. In vivo performance of common average referencing (CAR).

Suppl. Fig. 7. Noise immunity with common average referencing (CAR)

Suppl. Fig. 8. Subject S6 histology and accuracy by whisker

Suppl. Fig. 9. Resultant vector variance.

Suppl. Fig. 10. Subject S6, S8, S9 histology and directional curves

Suppl. Movie 1: GIF of growing electrode size

Suppl. Movie 2: MP3 of monopole, dipole orientation 


\section{Suppl. Table 1. LDA Classification Accuracy by Device and Electrode Configuration}

\begin{tabular}{|c|c|c|c|c|c|c|c|c|c|c|c|}
\hline \multirow[b]{2}{*}{ Device } & \multirow{2}{*}{$\begin{array}{c}\text { Electrode } \\
\text { Configuration } \\
\text { (Rows x } \\
\text { columns) }\end{array}$} & \multicolumn{9}{|c|}{ Subject Accuracy $(\%)^{c}$} & \multirow{2}{*}{$\begin{array}{c}\text { Average } \\
\text { Accuracy } \\
(\%)\end{array}$} \\
\hline & & S1 & S2 & S3 & S4 & S5 & S6 & S7 & S8 & S9 & \\
\hline \multirow{9}{*}{ DISC $^{a}$} & $16 \times 8$ & 90.2 & 98.6 & 99.1 & 95.9 & 94.0 & 93.2 & 91.5 & 99.2 & 95.9 & 95.3 \\
\hline & $16 \times 4$ & 92.5 & 98.2 & 98.2 & 95.4 & 91.3 & 90.9 & 89.9 & 97.7 & 94.7 & 94.3 \\
\hline & $11 \times 8$ & 92.7 & 98.4 & 98.5 & 96.3 & 92.3 & 93.5 & 91.6 & 98.8 & 95.7 & 95.3 \\
\hline & $11 \times 4$ & 93.8 & 97.7 & 92.3 & 95.5 & 89.3 & 91.4 & 89.8 & 97.7 & 94.7 & 93.6 \\
\hline & $3 \times 8$ & 92.8 & 97.1 & 84.9 & 93.8 & 88.9 & 88.7 & 86.5 & 97.8 & 92.0 & 91.4 \\
\hline & $3 \times 4$ & 92.7 & 94.5 & 81.5 & 91.8 & 87.3 & 85.9 & 83.6 & 95.4 & 91.1 & 89.3 \\
\hline & $1 \times 8$ & 57.8 & 82.0 & 61.9 & 85.9 & 72.1 & 79.4 & 82.6 & 96.1 & 85.3 & 78.1 \\
\hline & $1 \times 4$ & 57.3 & 57.1 & 48.0 & 85.0 & 68.0 & 75.6 & 77.9 & 91.4 & 80.8 & 71.2 \\
\hline & Best $1 \times 1^{b}$ & 32.7 & 30.6 & 38.2 & 64.3 & 42.6 & 59.3 & 30.4 & 56.6 & 50.2 & 45.0 \\
\hline \multirow{2}{*}{ Tetrodes } & Best 4 & 48.7 & 50.5 & 72.6 & 57.2 & 26.7 & 30.3 & 51.0 & 89.3 & 95.3 & 58.0 \\
\hline & Best 1 & 38.6 & 44.0 & 29.3 & 50.4 & 21.2 & 28.7 & 39.5 & 60.2 & 86.1 & 44.2 \\
\hline \multirow{2}{*}{$\begin{array}{l}\text { Virtual } \\
\text { Macro }\end{array}$} & $3 \times 400$ um & 35.8 & 63.7 & 57.4 & 81.6 & 51.1 & 63.5 & 65.6 & 69.6 & 54.4 & 60.3 \\
\hline & $2 \mathrm{~mm}$ & 25.1 & 41.5 & 37.5 & 73.8 & 22.4 & 41.0 & 25.1 & 34.7 & 31.6 & 37.0 \\
\hline
\end{tabular}

Note a: For the 11xn analysis, we selected 11 contiguous rows in the cortex, generally rows 111 or 2-12. Generally, the deepest rows showed little amplitude or variation. 3xn analysis selected 3 depths approximately inside layers II/III, V, and VI using depths from Paxinos, Watson $6^{\text {th }}$ Edition, Figure 52. $1 \mathrm{xn}$ analysis used layer $\mathrm{V}$ recordings. Each subject was adjusted slightly based on row 1 impedance and the location of a polarity change. Note b: Best channel was chosen as the channel in layer $\mathrm{V}$ with the highest amplitude. This electrode channel was fixed for each subject with no change by whisker. Note c: Accuracy after 10-fold crossvalidation. 
Suppl. Table 2. Source configuration table for a multi-source model (see Fig. 2).

\begin{tabular}{|c|c|r|r|}
\hline $\begin{array}{c}\text { Source } \\
\text { number }\end{array}$ & $\begin{array}{l}\text { Diameter, } \\
\varnothing_{\text {sh, } \mu \mathrm{m}}\end{array}$ & \multicolumn{2}{|l|}{$\begin{array}{l}\text { Gap } \\
\text { distance, mm }\end{array}$} \\
\hline 1 & 200 & 3.16 & 19 \\
\hline 2 & 400 & 0.6 & 94.4 \\
\hline 3 & 200 & 5.12 & 107 \\
\hline 4 & 800 & 1.73 & 190 \\
\hline 5 & 400 & 4.63 & 212 \\
\hline 6 & 800 & 3.93 & 345 \\
\hline 7 & 1600 & 5.0 & 349 \\
\hline 8 & 400 & & \\
\hline
\end{tabular}

Suppl. Table 2a. Electrical properties of electrostatic model (see Fig. 2).

\begin{tabular}{|c|c|c|c|c|c|c|}
\hline $\begin{array}{c}\text { Lead } \\
\text { Height } \\
(\mu \mathrm{m})\end{array}$ & $\begin{array}{c}\text { Lead } \\
\text { Conductivity } \\
(\mathrm{S} / \mathrm{m})\end{array}$ & $\begin{array}{c}\text { Brain } \\
\text { Conductivity } \\
(\mathrm{S} / \mathrm{m})\end{array}$ & $\begin{array}{c}\text { Electrode } \\
\text { Conductivity } \\
(\mathrm{S} / \mathrm{m})\end{array}$ & $\begin{array}{c}\text { Electrode } \\
\text { Pitch } \\
(\mu \mathrm{m})\end{array}$ & $\begin{array}{c}\text { Electrode } \\
\text { Radius } \\
(\mu \mathrm{m})\end{array}$ & $\begin{array}{c}\text { Current } \\
\text { Density, } \\
\mathrm{nA}- \\
\mathrm{m} / \mathrm{mm}^{2}\end{array}$ \\
\hline 6000 & $0.1 \mathrm{e}-9$ & 0.2 & $9.3 \mathrm{e} 6$ & 200 & 25 & 1.67 \\
& & & (platinum) & & (default) & \\
\hline
\end{tabular}




\section{Suppl. Table 3. Best Electrode SNR, Best Electrode Signal RMS, and Best Electrode Noise RMS of LFP band by Device}

\begin{tabular}{|c|c|c|c|c|c|c|c|c|c|c|c|}
\hline \multirow{2}{*}{ Device } & \multirow{2}{*}{$\begin{array}{l}\text { Electrode } \\
\text { Configuration } \\
\text { (Rows x } \\
\text { columns) }\end{array}$} & \multicolumn{8}{|c|}{ SNR LFP (dBV) } & \multirow[b]{2}{*}{ S9 } & \multirow{2}{*}{$\begin{array}{c}\text { Average } \\
\text { SNR }\end{array}$} \\
\hline & & $\mathrm{S} 1$ & S2 & S3 & S4 & S5 & S6 & S7 & S8 & & \\
\hline DISC & $11 \times 4$ & 10.8 & 16.8 & 14.6 & 13.2 & 10.0 & 12.3 & 10.3 & 14.2 & 14.8 & 12.6 \\
\hline DISC CAR & $11 \times 4$ & 13.9 & 18.3 & 15.9 & 14.5 & 13.7 & 15.3 & 15.5 & 15.2 & 15.5 & 15.3 \\
\hline Tetrodes & Best 4 & 8.0 & 12.3 & 7.5 & 10.4 & 5.8 & 5.1 & 6.8 & 7.6 & 7.3 & 7.7 \\
\hline \multirow{2}{*}{$\begin{array}{l}\text { Virtual } \\
\text { Macro }\end{array}$} & $2 \mathrm{~mm}$ & 3.8 & 12.2 & 8.8 & 11.1 & 4.0 & 4.9 & 2.8 & 8.5 & 9.8 & 7.1 \\
\hline & $3 \times 400$ um & 8.1 & 14.1 & 12.7 & 11.8 & 6.6 & 9.5 & 7.0 & 11.8 & 11.5 & 10.1 \\
\hline
\end{tabular}

\begin{tabular}{|c|c|c|c|c|c|c|c|c|c|c|c|}
\hline \multirow{2}{*}{ Device } & \multirow{2}{*}{$\begin{array}{l}\text { Electrode } \\
\text { Configuration } \\
\text { (Rows } \mathrm{x} \\
\text { columns) }\end{array}$} & \multicolumn{9}{|c|}{ RMS Signal (uVrms) } & \multirow{2}{*}{$\begin{array}{c}\text { Average } \\
\text { RMS } \\
\text { Signal }\end{array}$} \\
\hline & & $\mathrm{S} 1$ & $\mathrm{~S} 2$ & S3 & S4 & S5 & S6 & S7 & S8 & S9 & \\
\hline DISC & $11 \times 4$ & 20.7 & 73.1 & 46.8 & 73.1 & 10.9 & 25.4 & 12.3 & 29.0 & 41.4 & 35.2 \\
\hline DISC CAR & $11 \times 4$ & 18.8 & 61.3 & 42.4 & 32.4 & 9.4 & 22.3 & 11.3 & 24.8 & 33.5 & 27.8 \\
\hline Tetrodes & Best 4 & 24.1 & 56.3 & 28.9 & 18.7 & 10.1 & 19.5 & 20.5 & 28.7 & 42.1 & 25.3 \\
\hline \multirow{2}{*}{$\begin{array}{l}\text { Virtual } \\
\text { Macro }\end{array}$} & $2 \mathrm{~mm}$ & 9.3 & 41.3 & 18.5 & 67.9 & 6.1 & 11.7 & 4.2 & 13.8 & 28.7 & 21.5 \\
\hline & $3 \times 400$ um & 24.0 & 93.0 & 69.3 & 93.6 & 13.1 & 36.8 & 11.7 & 37.3 & 50.8 & 44.6 \\
\hline
\end{tabular}

\begin{tabular}{|c|c|c|c|c|c|c|c|c|c|c|c|}
\hline \multirow{2}{*}{ Device } & \multirow{2}{*}{$\begin{array}{l}\text { Electrode } \\
\text { Configuration } \\
\text { (Rows x } \\
\text { columns) }\end{array}$} & \multicolumn{9}{|c|}{ RMS Noise (uVrms) } & \multirow{2}{*}{$\begin{array}{c}\text { Average } \\
\text { RMS } \\
\text { Noise }\end{array}$} \\
\hline & & S1 & S2 & S3 & S4 & S5 & S6 & S7 & S8 & S9 & \\
\hline DISC & $11 \times 4$ & 4.0 & 4.5 & 4.5 & 7.2 & 2.4 & 4.9 & 2.7 & 3.1 & 4.5 & 4.1 \\
\hline DISC CAR & $11 \times 4$ & 2.4 & 3.6 & 3.7 & 3.5 & 1.1 & 2.6 & 1.1 & 2.6 & 2.9 & 2.6 \\
\hline Tetrodes & Best 4 & 4.6 & 4.5 & 5.9 & 2.5 & 3.4 & 6.0 & 4.3 & 5.4 & 9.2 & 5.4 \\
\hline
\end{tabular}




\begin{tabular}{|l|l|r|r|r|r|r|r|r|r|r|r|}
\hline $\begin{array}{l}\text { Virtual } \\
\text { Macro }\end{array}$ & $2 \mathrm{~mm}$ & 3.3 & 2.6 & 2.5 & 6.0 & 2.1 & 4.1 & 2.5 & 2.0 & 3.4 & 3.1 \\
\cline { 2 - 11 } & $3 \times 400 \mathrm{um}$ & 3.8 & 4.3 & 4.3 & 6.8 & 2.4 & 4.8 & 2.7 & 3.0 & 3.9 & 3.9 \\
\hline
\end{tabular}

Suppl. Table 4. LDA classification accuracy (\%) with different types of individual statistics estimated from gamma-band, DISC and tetrode

\begin{tabular}{|c|c|c|c|c|c|c|c|c|c|c|c|}
\hline \multirow[t]{3}{*}{ Device } & \multicolumn{10}{|c|}{ Subject-wise Accuracy (\%) } & \multirow{3}{*}{$\begin{array}{c}\text { Avg } \\
\text { Accuracy } \\
(\%)\end{array}$} \\
\hline & Methods & s1 & S2 & S3 & S4 & S5 & S6 & S7 & S8 & S9 & \\
\hline & & & & & & & & & & & \\
\hline \multirow[b]{5}{*}{ DISC } & ZC & 20.1 & 13.7 & 11.3 & 32.6 & 9.14 & 13.0 & 35.6 & 27.7 & 13.1 & 19.6 \\
\hline & RMS & 43.0 & 83.0 & 82.5 & 70.5 & 67.1 & 56.4 & 53.9 & 41.6 & 67.4 & 62.8 \\
\hline & Var & 41.4 & 56.5 & 72.3 & 66.7 & 56.5 & 35.7 & 44.6 & 31.7 & 56.5 & 51.3 \\
\hline & Phase & 12.6 & 61.5 & 32.4 & 27.1 & 35.6 & 43.3 & 16.6 & 38.9 & 19.9 & 31.9 \\
\hline & RV & 31.9 & 32.8 & 38.9 & 42.3 & 33.7 & 24.7 & 34.4 & 22.9 & 53.1 & 34.9 \\
\hline \multirow{4}{*}{ Tetrode } & ZC & 3.2 & 3.3 & 17.9 & 11.6 & 12.5 & 3.0 & 11.7 & 3.8 & 12.3 & 8.80 \\
\hline & RMS & 27.9 & 32.5 & 14.4 & 32.5 & 28.6 & 12.7 & 15.0 & 22.9 & 26.5 & 23.6 \\
\hline & Var & 27.1 & 31.6 & 20.3 & 22.6 & 11.6 & 12.5 & 14.3 & 21.8 & 17.2 & 19.8 \\
\hline & Phase & 25.1 & 37.9 & 11.6 & 4.27 & 13.6 & 12.2 & 19.3 & 29.6 & 13.2 & 18.5 \\
\hline
\end{tabular}

\section{Individual Statistical Method based Neural decoding:}

In another effort to study the contributing factors in decoding, a simplified feature matrix was created using the following scalars: Zero-crossing, RMS, variance, Phase at maximum peak of Fourier transform (FT), and resultant vector. Each was independently tested (Suppl. Fig. XX) for gamma band waveform (0:0.5:100 ms). Since, electrode configurations for 11 rows $x 8$ columns and 11 rows $x 4$ columns do not show significant difference of classification accuracy, by considering computational load and simplicity of system, we used 11 x 4 configuration to observe the performance of DISC. We performed 10-fold cross-validation with LDA such that each run 
serves once for validation. Finally, the recognition result with accuracy was taken by averaging all the runs. The method RMS shows significant outperform compared to others (Avg accuracy: 62.84, $p<0.05$ shown in Table 4). The short review of statistical methods is the following:

Zero-Crossing (ZC): The zero-crossing $(\mathrm{ZC})$ is the number of sign changes along a Gamma LFP signal, defined as:

$$
Z C=\left\{\begin{array}{l}
1, x_{i} \cdot x_{i+1}<0 \\
0, \text { Otherwise }
\end{array}\right.
$$

where $x_{i}$ and $x_{i+1}$ are the two consecutive data points from a Gamma LFP signal.

Root-Mean Square (RMS): The root mean square (RMS) is amplitude from gamma LFP signals over 0-100 ms period:

$$
R M S=\sqrt{\frac{1}{N} \sum_{i=1}^{N} x_{i}^{2}}
$$

where $N$ denotes the length of the signals $x$ for each gamma band waveform.

Variance (Var): The variance (Var) of signal refers to a statistical measuring how the amplitudes of the LFP signals for each whisker are spread out from their average value, and is also used to characterize the nature of each whisker LFP signals defined as:

$$
\operatorname{Var}=\frac{1}{N-1} \sum_{i=1}^{N}\left(x_{i}-\mu\right)^{2}
$$

where $N$ and $\mu$ represent the number of sample points and mean respectively from gamma band signal $x$.

Phase: In signal processing applications, the phase of signals is another key feature like amplitude analysis for identifying whisker activity, using the following approach was used:

$$
\varnothing=\tan ^{-1}\left(\frac{X_{\text {im }}}{Y_{\text {real }}}\right)
$$

where $X_{i m}$ and $X_{\text {real }}$ are the real and imagery parts of Fourier Transform (FT) at a maximum energy.

Resultant Vector (RV): For machine-learning application with DISC device, a set of vectors was estimated from 8 electrode columns at layer $V$ for each trial and summing up to represent the resultant vectors. A simplified feature matrix is created based on the estimated resultant vector. 


\section{Suppl. Table 5. Review of Recent Safety and Efficacy Differences in Epileptogenic Diagnosis using ECoG vs SEEG}

\begin{tabular}{|c|c|c|c|c|c|c|}
\hline Reference & $\begin{array}{l}\text { Number } \\
\text { of } \\
\text { Patients }\end{array}$ & Hemorrhagic & Infections & $\begin{array}{l}\text { CSF } \\
\text { leakage }\end{array}$ & $\begin{array}{l}\text { Seizure } \\
\text { Free }\end{array}$ & Notes \\
\hline $\begin{array}{l}\text { Jehi et al, } 2021 \\
\text { Ann. Neurol. }\end{array}$ & $\begin{array}{l}502 \text { SDE } \\
903 \text { SEEG }\end{array}$ & $\begin{array}{l}1.8 \% \\
1.6 \%\end{array}$ & $\begin{array}{l}7.0 \% \\
0.9 \%\end{array}$ & & $\begin{array}{l}41.1 \% \\
54.6 \%\end{array}$ & $\begin{array}{l}10 \text { sites across } 7 \\
\text { countries }\end{array}$ \\
\hline $\begin{array}{l}\text { Tandon et al, } 2019 \\
\text { JAMA }\end{array}$ & $\begin{array}{l}139 \text { SDE } \\
121 \text { SEEG }\end{array}$ & $\begin{array}{l}5.0 \% \\
0\end{array}$ & $\begin{array}{l}2.2 \% \\
0\end{array}$ & & $\begin{array}{l}55 \% \\
76 \%\end{array}$ & $\begin{array}{l}\text { Single site, } \sim 6 \text { years. } \\
\text { Even though } 71 \% \text { vs } 44 \% \\
\text { of patients were lesional, } \\
\text { SEEG still outperformed } \\
\text { SDE. }\end{array}$ \\
\hline $\begin{array}{l}\text { Joswig, et al } 2020 \\
\text { Neurosurgery }\end{array}$ & $\begin{array}{l}355 \text { SDE } \\
145 \text { SEEG }\end{array}$ & $\begin{array}{l}1.4 \% \\
2.8 \%\end{array}$ & $\begin{array}{l}2.3 \% \\
0 \%\end{array}$ & & & Same OR time \\
\hline $\begin{array}{l}\text { Yan et al, } 2019 \\
\text { Epilepsia }\end{array}$ & $\begin{array}{l}2,036 \text { SDE } \\
1,973 \text { SEEG }\end{array}$ & $\begin{array}{l}4.8 \% \\
4.4 \%, p=0.001\end{array}$ & $\begin{array}{l}1.6 \% \\
0.9 \%\end{array}$ & $\begin{array}{l}0.6 \% \\
0 \%\end{array}$ & $\begin{array}{l}56 \% \\
61 \%\end{array}$ & $\begin{array}{l}\text { Med complications: } 2.6 \\
\text { vs } 0.7 \% \\
\text { Transient deficit: } 5.7 \text { vs } \\
1.9 \%, p=.01 \\
\text { Length of stay } 8.9 \text { vs } 6.3 \\
\text { days (NS) }\end{array}$ \\
\hline $\begin{array}{l}\text { Sacino et al, } 2019 \\
\text { Clinical Neurosurg }\end{array}$ & $\begin{array}{l}697 \text { SDE } \\
277 \text { SEEG }\end{array}$ & $\begin{array}{l}10.7 \% \\
2.9 \%\end{array}$ & $\begin{array}{l}10.8 \% \\
0 \%\end{array}$ & $\begin{array}{l}11.9 \% \\
0.3 \%\end{array}$ & $\begin{array}{l}52.1 \% \\
66.5 \%\end{array}$ & $\begin{array}{l}\text { Children only. One SEEG } \\
\text { mortality. None for SDE. }\end{array}$ \\
\hline $\begin{array}{l}\text { Toth, et al. } 2019 \\
\text { Seizure }\end{array}$ & $\begin{array}{l}1025 \text { SDE } \\
974 \text { SEEG }\end{array}$ & & & & $\begin{array}{l}55.9 \% \\
64.7 \%\end{array}$ & $\begin{array}{l}\text { Even better seizure-free if } \\
\text { resection was in temporal } \\
\text { lobe ( } 57 \text { vs } 72 \% \text { ) }\end{array}$ \\
\hline $\begin{array}{l}\text { Yang, et. al. } 2017 \\
\text { Stereotactic \& } \\
\text { Functional } \\
\text { Neurosurg }\end{array}$ & $\begin{array}{l}52 \text { SDE } \\
48 \text { SEEG }\end{array}$ & \multicolumn{2}{|c|}{$\begin{array}{l}17.3 \% \\
4.2 \%\end{array}$} & & $\begin{array}{l}52.2 \% \\
59.5 \%\end{array}$ & $\begin{array}{l}\text { Complication rate was } \\
25 \% \text { vs } 16.7 \%\end{array}$ \\
\hline
\end{tabular}

Providing safer surgery is a major advantage of our approach. Suppl. Table 5 lists 7 retrospective reviews comparing SEEG to ECoG implants. A reduced risk of infection was found in 6 of 6 studies and a reduction of hemorrhaging in 5 of 6 . SEEG allows surgeons to reduce infection from 4.1 to $0.8 \%$ and hemorrhaging from 5.2 to $3.3 \%$ (weighted average by population size), which does not include the Yang study. 

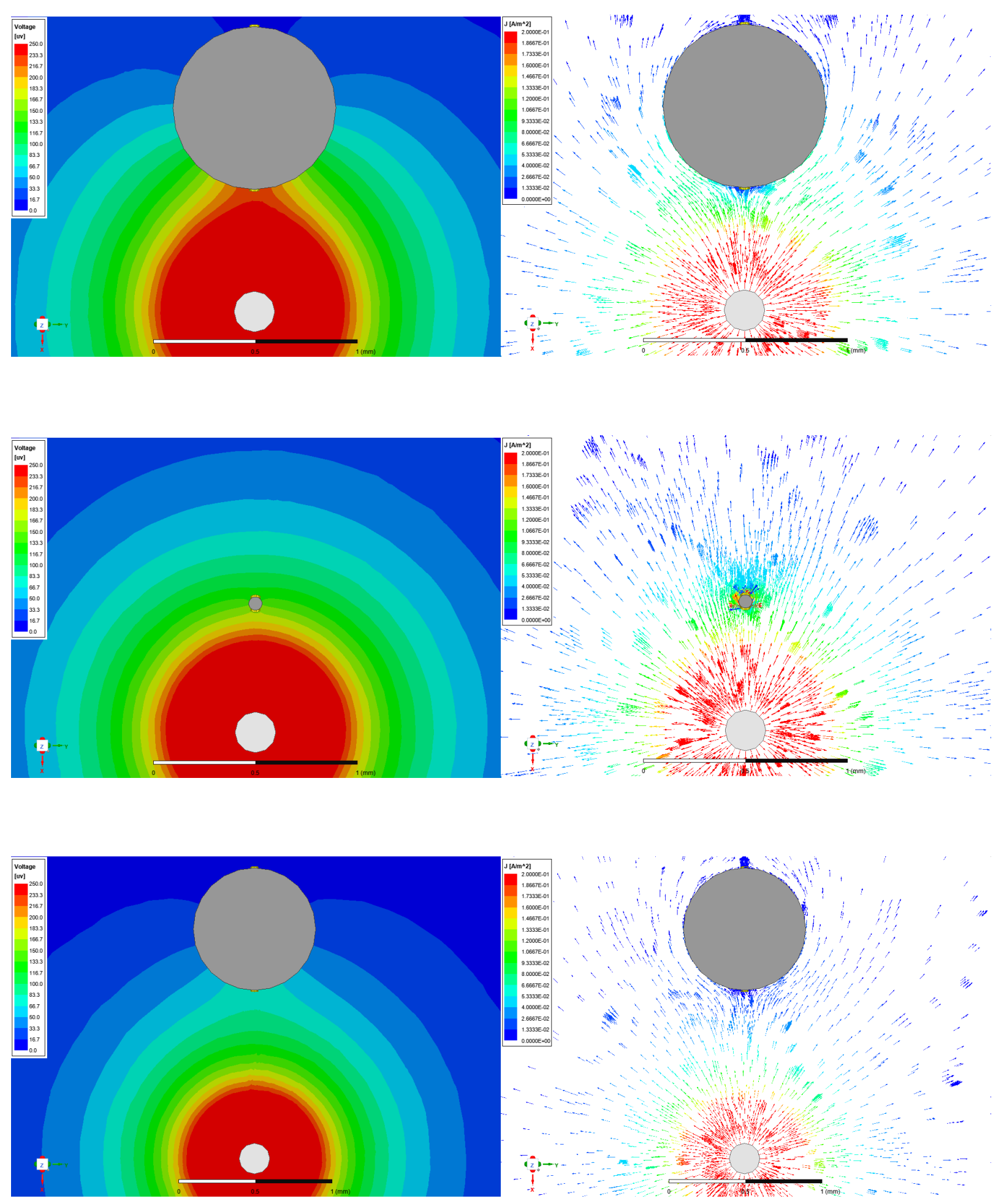

Suppl. Fig. 1: 2D Voltage contour and J-field illustrating the mechanism of substrate shielding. Top-down view of a current field and potential contours around insulating bodies. 


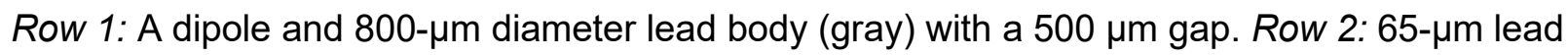
body (microwire-sized device) with a $500 \mu \mathrm{m}$ gap. Row 3: 800- $\mu \mathrm{m}$ diameter lead body with 1$\mathrm{mm}$ gap. The relative positions and scales are kept constant in rows 1 and 2 .

The current vectors (right) illustrate the rapid divergence around the insulating body compared to microwire-sized array (row 2). The potential contour lines (left) are orthogonal to the J-field. The voltage gradient (density of contour lines) is unchanged where the change is current is near zero. Thus, an isopotential extends away from the electrode toward the source in response to a static J-field. Similarly, an isopotential extends away from the opposite side electrode and has a much lower magnitude. In summary, DISC shows greater directionality (potential differences) as the device diameter increases, directing more current flow around the lead body shaft and amplifying the difference in front and back electrode potential.

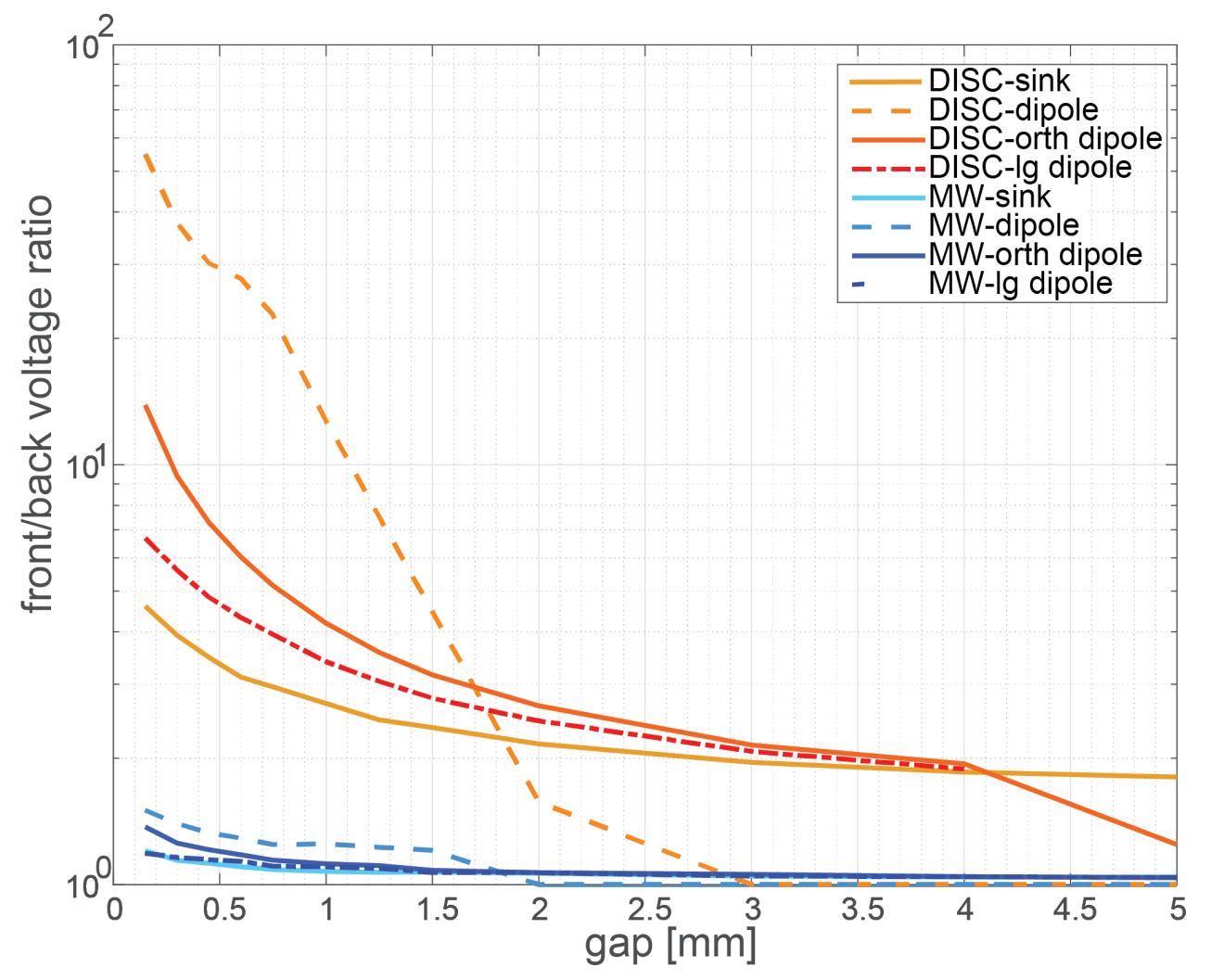

\section{Suppl. Fig. 2. Front/back amplitude by source type and orientation (related to Suppl.}

Movie 2). FEM results for a $800-\mu \mathrm{m}$ insulating body and a $200-\mu \mathrm{m}$ dipole over a gap distance of $5 \mathrm{~mm}$. The parallel-oriented dipole has the greatest directionality but the least range $(3 \mathrm{~dB}$ 
distance equals $1.9 \mathrm{~mm}$ ). The monopole and orthogonal dipole performed similarly with a $3 \mathrm{~dB}$ distance equal to $4.0 \mathrm{~mm}$ ) (see also Suppl. Movie 2).

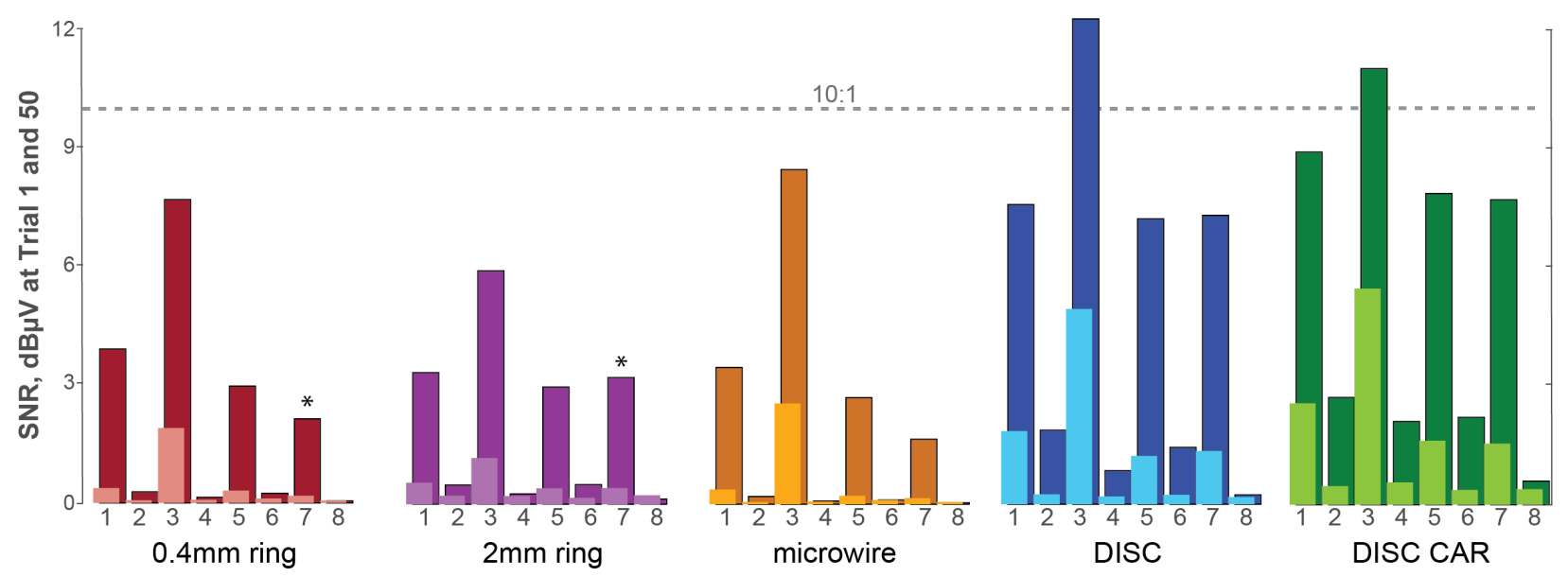

Suppl. Fig 3. SNR results for an 8-source model with a 2-mm ring electrode. The FEM model described in Fig. 2 was also analyzed with a 2-mm tall ring electrode (standard size of an SEEG). Relative to the $0.4-\mathrm{mm}$-tall ring, this ring SNR was $1-2 \mathrm{~dB} \mu \mathrm{V}$ or $25-50 \%$ lower in the typical case, and in the case of source 7 (large, distant neural source), the $2 \mathrm{~mm}$ ring performs slightly better. This is because biological noise is attenuated much more for the larger ring, leading to better SNR values in the presence of high amplitude noise. To ensure fair comparison, the center of the ring was always fixed to the same plane as the sink.

$\left(^{*}\right)$ Due to the higher SNR value for source 7, further analysis was done in Suppl. Fig. 4 to explore the properties of the different ring sizes. 

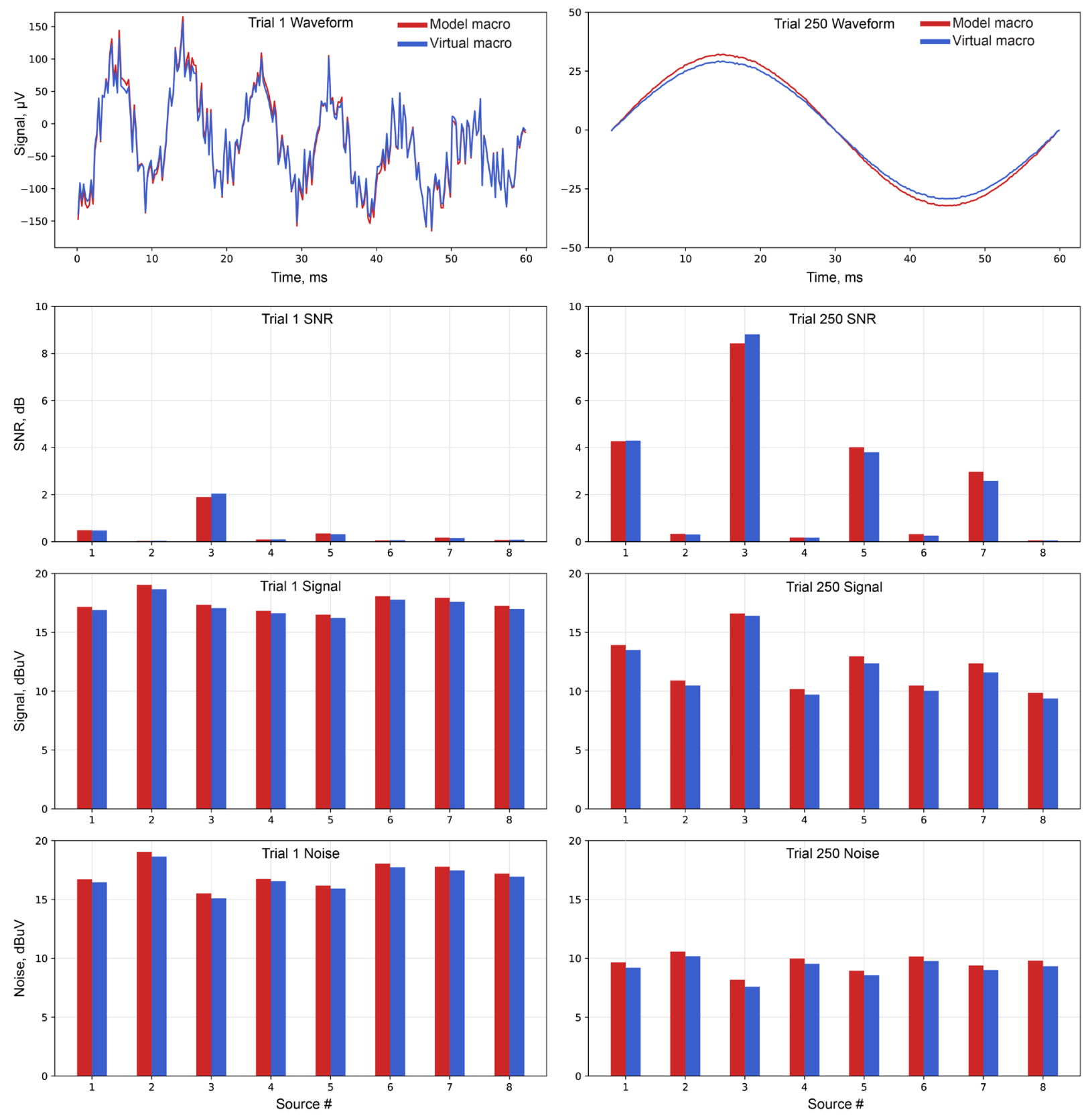

Suppl. Fig. 4. SNR, amplitude, and noise comparison between a FEM ring and virtual DISC ring electrode. An ANSYS model of a 0.4-mm tall ring electrode was tested in an 8source environment (Fig. 2). This was compared with a 3 row, 8 column microelectrode array $(200 \mu \mathrm{m}$ vertical pitch) in the same location then digitally averaged. The SNR, amplitude, and noise are separated for comparison. The left column represents one randomly seeded trial, and the right is the average after 250 trials. The ring was assigned $2.7 \mu \mathrm{Vrms}$ while each microelectrode was assigned $4.3 \mu \mathrm{Vrms}$, which represented the average noise floor values. 
Both devices were modeled using Suppl. Table 2 and 2a, the same parameters as Fig. 2. SNR, RMS noise and RMS signal were calculated using equations 4,2 , and 3 , respectively. These results indicate a deviation of no more than $0.5 \mathrm{~dB}$ for any measurement. Source phase and electronic noise were kept consistent between models for trial 1 to fairly compare, but the remaining 249 trials were randomized. Since the right column is all 250 trials averaged, it is not necessary to fix the phase, frequency, and noise of the models.

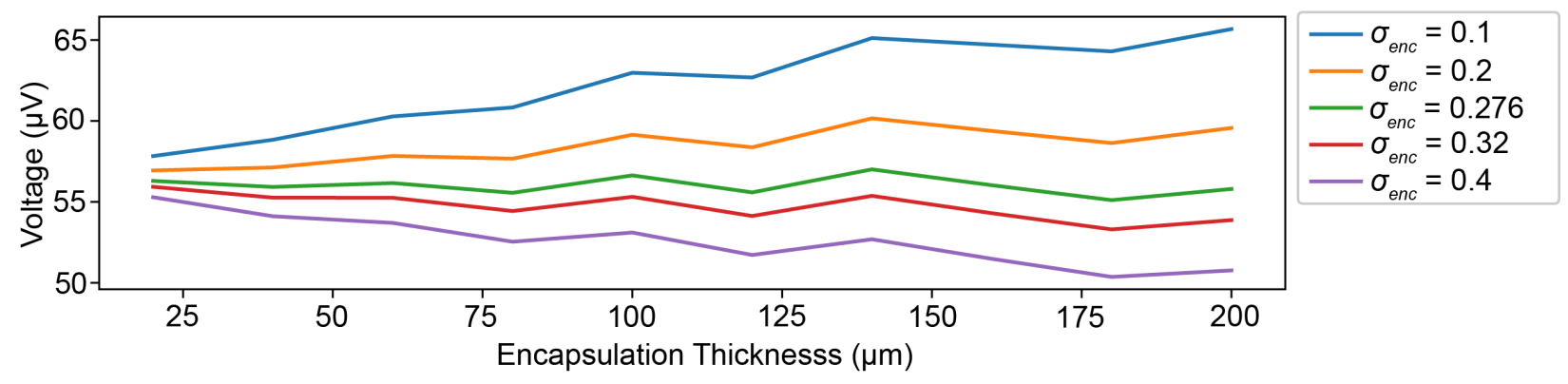

\section{Suppl. Fig. 5. ANSYS model of encapsulation around $800-\mu \mathrm{m}$ diameter lead body}

Encapsulation thickness around the lead body was 0-200 microns thick and $\sigma_{\text {enc }} 0.1,0.2,0.276$, 0.32 , and $0.4 \mathrm{~S} / \mathrm{m}$. In this analysis, brain conductivity used was $0.27 \mathrm{~S} / \mathrm{m}$. Even with a $0.4 \mathrm{~S} / \mathrm{m}$ edemic layer, the amplitude reduction is less than $10 \%$. While seemingly contradictory, encapsulation tissue amplifies the measured voltage as predicted originally by Moffitt, Mclntyre 2005 (Moffitt and Mclntyre 2005). As discussed in Suppl. Fig. 1, the voltage gradient (density of contour lines) is unchanged where the change is current is near zero. Encapsulation tissue in this range and at this thickness creates an extended isopotential in front of the electrode in response to a static J-field.

The model used for this analysis is identical to the previous, with the addition of a cylindrical conductive layer around the device with the electrodes. 

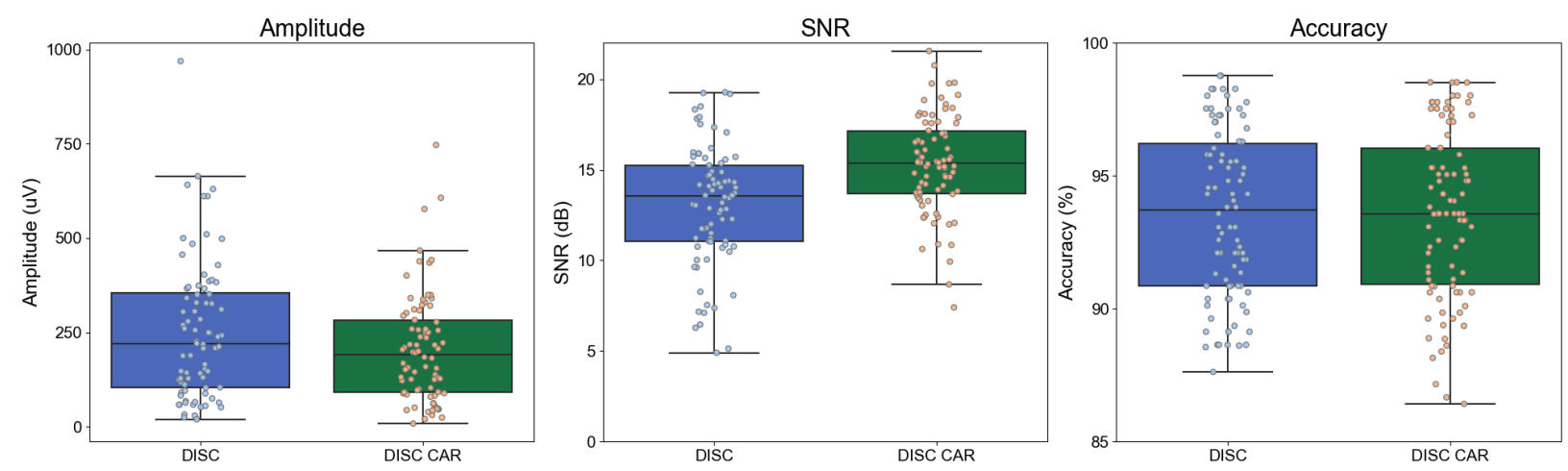

Suppl. Fig. 6. In vivo performance of common average referencing (CAR). ( $N=9$ subjects, 9 whiskers each). Amplitude and SNR data points represent the average by whisker and subject. Each accuracy value is the 10 -fold cross-validation for the 9-whisker model by subject. Given noise was low due to shielding from a Faraday cage, the advantage of CAR is negligible. These results will vary considerably given environmental factors.

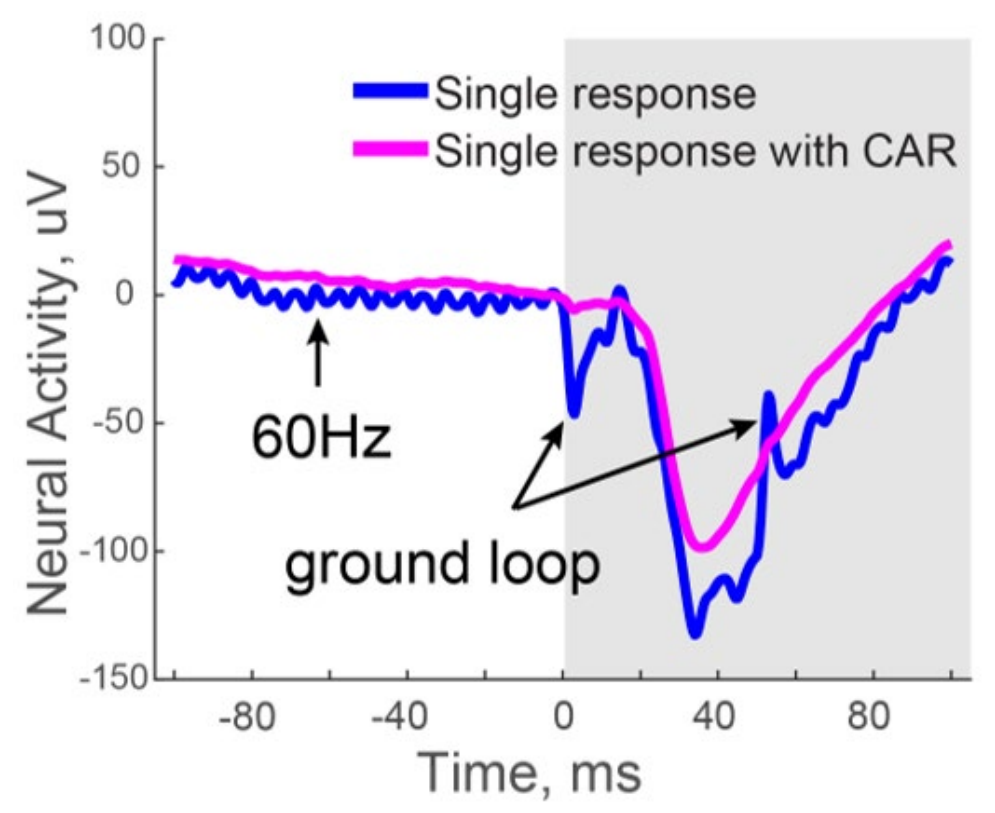

Suppl. Fig. 7. Noise immunity with common average referencing (CAR). Sample single trial of DISC in an in vivo stimulation trial without the use of a Faraday cage. The ground loop was intentionally created by connecting the air puffer ground at a separate outlet. Both signals were identically filtered. CAR effectively removes common mode noise with a small decrease in the evoked potential. 

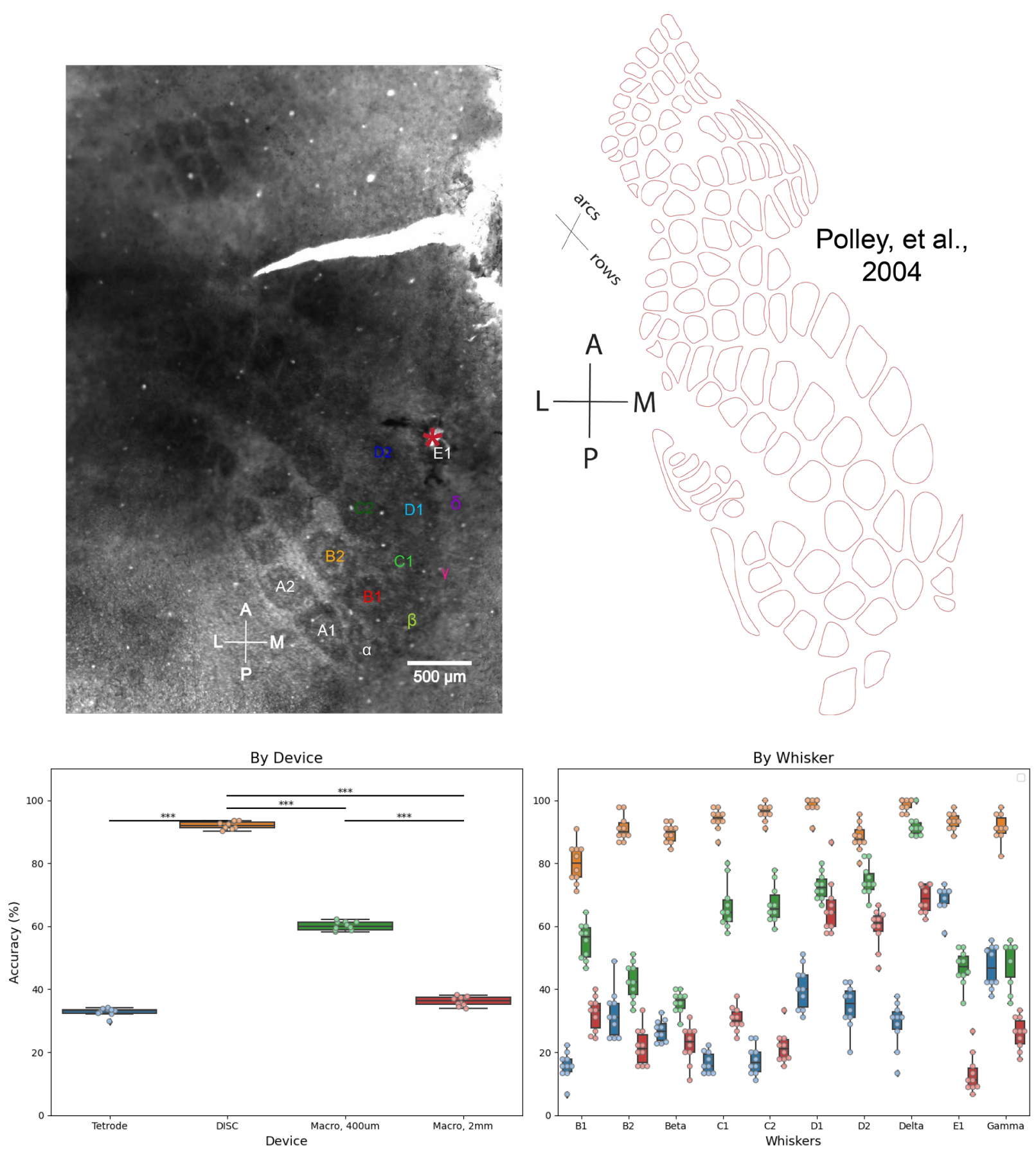

Suppl. Fig. 8. Subject $\mathbf{S} 6$ histology and accuracy by whisker. The position of the implant was barrel E1. A useful reference for identifying barrels in rats is Polley et al. (Polley, Kvašňák, and Frostig 2004). Further evidence of implanting in E1 was the tetrode amplitude, which was maximum at E1 (data not shown). DISC performed well in classification across the whiskers despite a maximum distance of $1.45 \mathrm{~mm}$. Also promising is the overall performance even though most sources were positioned at roughly the same angle from DISC. 

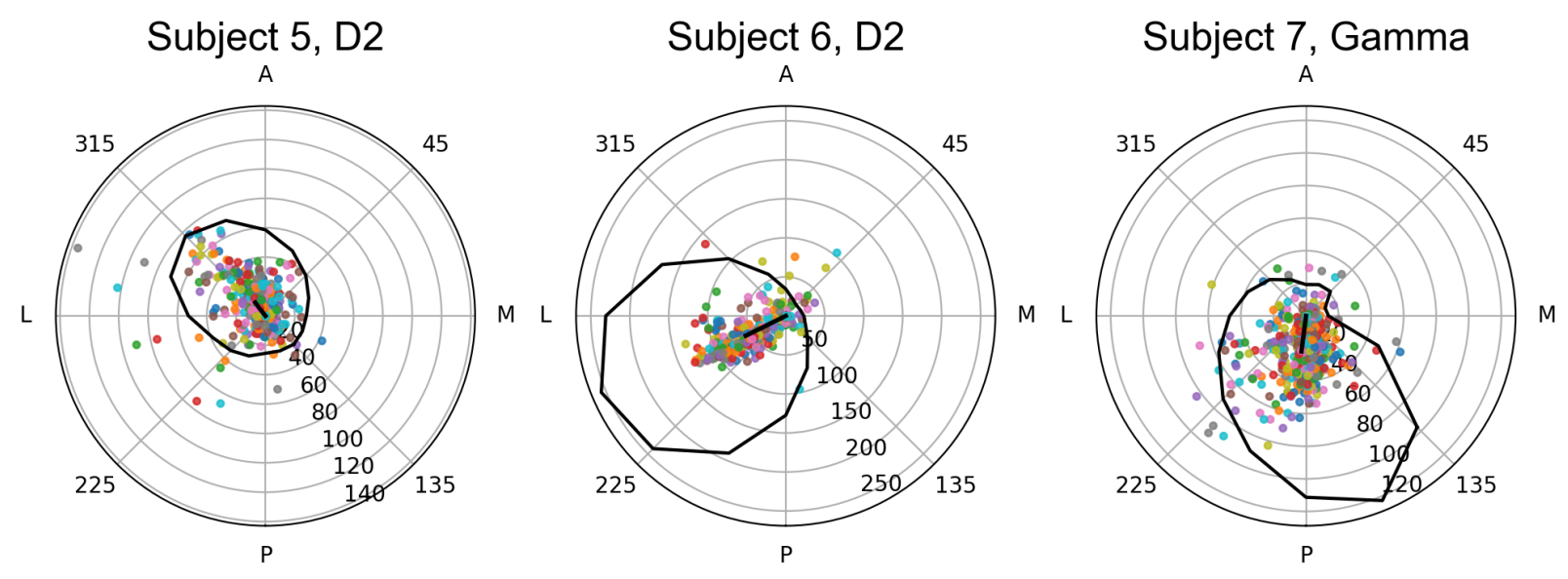

Suppl. Fig. 9. Resultant vector variance. Each colored dot represents the resultant vector for 450 trials for these example whiskers and subjects. The mean resultant vector (black line) and average direction curve illustrate the trend of the data (black closed circle, minimum value subtracted to improve contrast). 

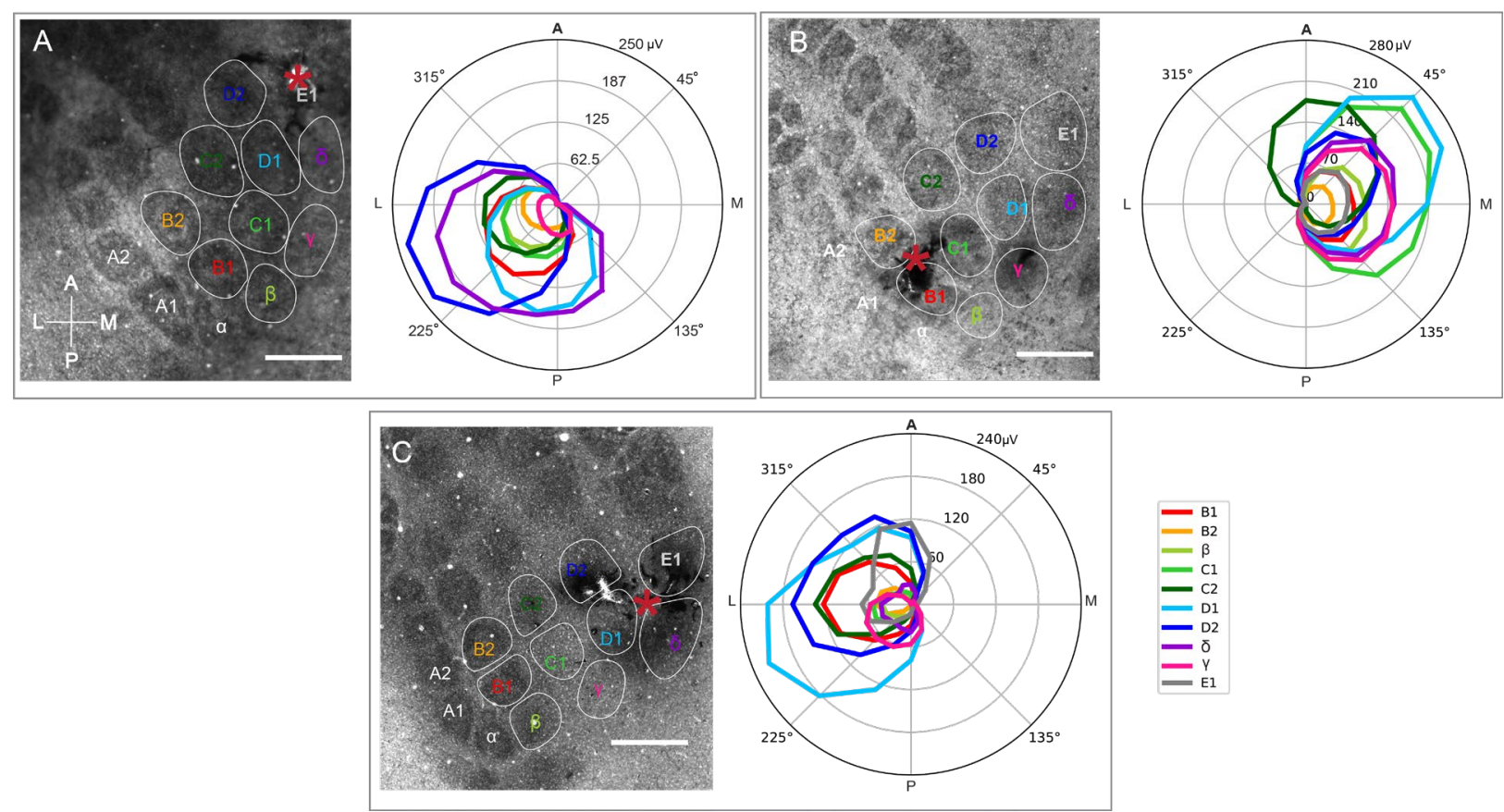

Suppl. Fig. 10. Subject S6, S8, S9 histology and directional curves. The results demonstrate close agreement with histology and the individual whisker response. Directional curve taken from layer $\mathrm{V}$ electrode ring.

(Movie 1 \& Movie 2 are not shareable on bioRxiv.) 


\section{Bibliography}

Anderson, David J, Karim G Oweiss, and Steven M Bierer. 2001. "Sensor Arrays in the MicroEnvironment of the Brain." In Acoustics, Speech, and Signal Processing, 2001. Proceedings.(ICASSP'01). 2001 IEEE International Conference On, 6:3433-36. IEEE.

Angrick, M., M. Ottenhoff, L. Diener, D. Ivucic, G. Ivucic, S. Goulis, J. Saal, et al. 2020. "RealTime Synthesis of Imagined Speech Processes from Minimally Invasive Recordings of Neural Activity." BioRxiv, no. 2. https://doi.org/10.1101/2020.12.11.421149.

Baillet, Sylvain, John C Mosher, and Richard M Leahy. 2001. "Electromagnetic Brain Mapping." IEEE Signal Processing Magazine 18 (6): 14-30.

Bancaud, Jean, Jean Talairach, A Bonis, C Schaub, G Szikla, P Morel, and M Bordas-Ferrer. 1965. “La Stéreoencephalographie Dans l'épilepsie." Mattson, Paris, 113-46.

Blanche, Timothy J, Martin A Spacek, Jamille F Hetke, and Nicholas V Swindale. 2005. "Polytrodes: High-Density Silicon Electrode Arrays for Large-Scale Multiunit Recording." Journal of Neurophysiology 93 (5): 2987-3000.

Bullard, Autumn J., Brianna C. Hutchison, Jiseon Lee, Cynthia A. Chestek, and Parag G. Patil. 2020. "Estimating Risk for Future Intracranial, Fully Implanted, Modular Neuroprosthetic Systems: A Systematic Review of Hardware Complications in Clinical Deep Brain Stimulation and Experimental Human Intracortical Arrays." Neuromodulation 23 (4): 41126. https://doi.org/10.1111/ner.13069.

Buzsáki, György, Costas A. Anastassiou, and Christof Koch. 2012. "The Origin of Extracellular Fields and Currents-EEG, ECoG, LFP and Spikes." Nature Reviews Neuroscience 13 (6): 407-20. https://doi.org/10.1038/nrn3241.

Du, Jiangang, Michael L Roukes, and Sotiris C Masmanidis. 2009. "Dual-Side and ThreeDimensional Microelectrode Arrays Fabricated from Ultra-Thin Silicon Substrates." Journal of Micromechanics and Microengineering 19 (7): 75008.

Dura-Bernal, Salvador, Benjamin A. Suter, Padraig Gleeson, Matteo Cantarelli, Adrian Quintana, Facundo Rodriguez, David J. Kedziora, et al. 2019. “NetpyNE, a Tool for DataDriven Multiscale Modeling of Brain Circuits." ELife 8: 1-26. https://doi.org/10.7554/eLife.44494.

Engel, Jerome. 2018. "The Current Place of Epilepsy Surgery." Current Opinion in Neurology 31 
(2): 192-97. https://doi.org/10.1097/WCO.0000000000000528.

Flint, Robert D, Zachary A Wright, Michael R Scheid, and Marc W Slutzky. 2013. "Long Term, Stable Brain Machine Interface Performance Using Local Field Potentials and Multiunit Spikes." Journal of Neural Engineering 10 (5): 56005.

Fried, Itzhak, Charles L. Wilson, Nigel T. Maidment, Jerome Engel, Eric Behnke, Tony A. Fields, Katherine A. Macdonald, Jack W. Morrow, and Larry Ackerson. 1999. "Cerebral Microdialysis Combined with Single-Neuron and Electroencephalographic Recording in Neurosurgical Patients: Technical Note." Journal of Neurosurgery 91 (4): 697-705. https://doi.org/10.3171/jns.1999.91.4.0697.

Gerbella, M., E. Borra, F. Pothof, M. Lanzilotto, A. Livi, L. Fogassi, O. Paul, G. A. Orban, P. Ruther, and L. Bonini. 2021. "Histological Assessment of a Chronically Implanted Cylindrically-Shaped, Polymer-Based Neural Probe in the Monkey." Journal of Neural Engineering 18 (2). https://doi.org/10.1088/1741-2552/abdd11.

Hagen, Espen, Solveig Næss, Torbjørn V. Ness, and Gaute T. Einevoll. 2018. "Multimodal Modeling of Neural Network Activity: Computing LFP, ECoG, EEG, and MEG Signals with LFPy 2.0." Frontiers in Neuroinformatics 12 (December). https://doi.org/10.3389/fninf.2018.00092.

Hämäläinen, Matti, Riitta Hari, Risto J Ilmoniemi, Jukka Knuutila, and Olli V Lounasmaa. 1993. "Magnetoencephalography-Theory, Instrumentation, and Applications to Noninvasive Studies of the Working Human Brain." Reviews of Modern Physics 65 (2): 413.

Hammer, Jiří, Tobias Pistohl, Jörg Fischer, Pavel Kršek, Martin Tomášek, Petr Marusič, Andreas Schulze-Bonhage, Ad Aertsen, and Tonio Ball. 2016. "Predominance of Movement Speed over Direction in Neuronal Population Signals of Motor Cortex: Intracranial EEG Data and A Simple Explanatory Model." Cerebral Cortex 26 (6): 2863-81. https://doi.org/10.1093/cercor/bhw033.

Herff, Christian, Dean J. Krusienski, and Pieter Kubben. 2020. "The Potential of StereotacticEEG for Brain-Computer Interfaces: Current Progress and Future Directions." Frontiers in Neuroscience 14 (February): 1-8. https://doi.org/10.3389/fnins.2020.00123.

Herreras, Oscar. 2016. "Local Field Potentials: Myths and Misunderstandings." Frontiers in Neural Circuits 10 (DEC): 1-16. https://doi.org/10.3389/fncir.2016.00101. 
Hochberg, Leigh R, Daniel Bacher, Beata Jarosiewicz, Nicolas Y Masse, John D Simeral, Joern Vogel, Sami Haddadin, Jie Liu, Sydney S Cash, and Patrick van der Smagt. 2012. "Reach and Grasp by People with Tetraplegia Using a Neurally Controlled Robotic Arm." Nature 485 (7398): 372-75.

Jehi, Lara, Marcia Morita-Sherman, Thomas E Love, Fabrice Bartolomei, William Bingaman, Kees Braun, Robyn Busch, John Duncan, Walter J Hader, and Guoming Luan. 2021. "Comparative Effectiveness of Stereo-EEG versus Subdural Grids in Epilepsy Surgery." Annals of Neurology.

Jun, James J, Nicholas A Steinmetz, Joshua H Siegle, Daniel J Denman, Marius Bauza, Brian Barbarits, Albert K Lee, Costas A Anastassiou, Alexandru Andrei, and Çağatay Aydın. 2017. "Fully Integrated Silicon Probes for High-Density Recording of Neural Activity." Nature 551 (7679): 232.

Kaiboriboon, Kitti, Ayham M Malkhachroum, Ahmad Zrik, Ahmad Daif, Nicholas M Schiltz, David M Labiner, and Samden D Lhatoo. 2015. "Epilepsy Surgery in the United States: Analysis of Data from the National Association of Epilepsy Centers." Epilepsy Research 116: 105-9.

Kajikawa, Yoshinao, and Charles E. Schroeder. 2011. "How Local Is the Local Field Potential?" Neuron 72 (5): 847-58. https://doi.org/10.1016/j.neuron.2011.09.029.

_ 2015. "Generation of Field Potentials and Modulation of Their Dynamics through Volume Integration of Cortical Activity." Journal of Neurophysiology 113 (1): 339-51. https://doi.org/10.1152/jn.00914.2013.

Kato, Hiroyuki K, Samuel K Asinof, and Jeffry S Isaacson. 2017. "Network-Level Control of Frequency Tuning in Auditory Cortex." Neuron 95 (2): 412-23.

Koessler, Laurent, Sophie Colnat-Coulbois, Thierry Cecchin, Janis Hofmanis, Jacek P Dmochowski, Anthony M Norcia, and Louis G Maillard. 2017. "In-vivo Measurements of Human Brain Tissue Conductivity Using Focal Electrical Current Injection through Intracerebral Multicontact Electrodes." Human Brain Mapping 38 (2): 974-86.

Kovac, Stjepana, Vejay N Vakharia, Catherine Scott, and Beate Diehl. 2017. "Invasive Epilepsy Surgery Evaluation." Seizure 44: 125-36.

Lauer, Simon M, Undine Schneeweiß, Michael Brecht, and Saikat Ray. 2018. "Visualization of Cortical Modules in Flattened Mammalian Cortices." Journal of Visualized Experiments: 
JoVE, no. 131.

Lindén, Henrik, Tom Tetzlaff, Tobias C. Potjans, Klas H. Pettersen, Sonja Grün, Markus Diesmann, and Gaute T. Einevoll. 2011. "Modeling the Spatial Reach of the LFP." Neuron 72 (5): 859-72. https://doi.org/10.1016/j.neuron.2011.11.006.

Luan, Lan, Xiaoling Wei, Zhengtuo Zhao, Jennifer J. Siegel, Ojas Potnis, Catherine A. Tuppen, Shengqing Lin, et al. 2017. "Ultraflexible Nanoelectronic Probes Form Reliable, Glial ScarFree Neural Integration." Science Advances 3 (2): 1-10.

https://doi.org/10.1126/sciadv.1601966.

Lucka, Felix, Sampsa Pursiainen, Martin Burger, and Carsten H Wolters. 2012. "Hierarchical Bayesian Inference for the EEG Inverse Problem Using Realistic FE Head Models: Depth Localization and Source Separation for Focal Primary Currents." Neurolmage 61 (4): 1364-82.

Ludwig, Kip A, Rachel M Miriani, Nicholas B Langhals, Michael D Joseph, David J Anderson, and Daryl R Kipke. 2009. "Using a Common Average Reference to Improve Cortical Neuron Recordings from Microelectrode Arrays." Journal of Neurophysiology 101: 1679_ 89. https://doi.org/10.1152/jn.90989.2008.

Makin, Joseph G, David A Moses, and Edward F Chang. 2020. "With an Encoder - Decoder Framework." Nature Neuroscience 23 (4): 575-82. https://doi.org/10.1038/s41593-0200608-8.

Moffitt, Michael A, and Cameron C Mclntyre. 2005. "Model-Based Analysis of Cortical Recording with Silicon Microelectrodes." Clinical Neurophysiology 116 (9): 2240-50.

Moses, David A., Sean L. Metzger, Jessie R. Liu, Gopala K. Anumanchipalli, Joseph G. Makin, Pengfei F. Sun, Josh Chartier, et al. 2021. "Neuroprosthesis for Decoding Speech in a Paralyzed Person with Anarthria." New England Journal of Medicine 385 (3): 217-27. https://doi.org/10.1056/nejmoa2027540.

Murakami, Shingo, and Yoshio Okada. 2015. "Neurolmage Invariance in Current Dipole Moment Density across Brain Structures and Species : Physiological Constraint for Neuroimaging." Neurolmage 111: 49-58. https://doi.org/10.1016/j.neuroimage.2015.02.003.

Na, Kyounghwan, Zachariah J Sperry, Jiaao Lu, Mihaly Voeroeslakos, Saman S Parizi, Tim M 
Bruns, Euisik Yoon, and John P Seymour. "Novel Diamond Shuttle to Deliver Flexible Bioelectronics with Reduced Tissue Compression." Microsystems \& Nanoengineering, January. http://biorxiv.org/content/early/2018/10/17/435800.abstract.

Næss, Solveig, Geir Halnes, Espen Hagen, Donald J Hagler, Anders M Dale, Gaute T Einevoll, and Torbjørn V Ness. 2021. "Biophysically Detailed Forward Modeling of the Neural Origin of EEG and MEG Signals.” Neurolmage 225 (September 2020): 117467. https://doi.org/10.1016/j.neuroimage.2020.117467.

Neymotin, Samuel A., Dylan S. Daniels, Blake Caldwell, Robert A. McDougal, Nicholas T. Carnevale, Mainak Jas, Christopher I. Moore, Michael L. Hines, Matti Hämäläinen, and Stephanie R. Jones. 2020. "Human Neocortical Neurosolver (HNN), a New Software Tool for Interpreting the Cellular and Network Origin of Human MEG/EEG Data." ELife 9: 1-39. https://doi.org/10.7554/eLife.51214.

Noor, M Sohail, and Cameron C Mcintyre. 2021. "Biophysical Characterization of Local Field Potential Recordings from Directional Deep Brain Stimulation Electrodes." Clinical Neurophysiology. https://doi.org/10.1016/j.clinph.2021.01.027.

Nunez, Paul L., and Ramesh Srinivasan. 2010. "Scale and Frequency Chauvinism in Brain Dynamics: Too Much Emphasis on Gamma Band Oscillations." Brain Structure and Function 215 (2): 67-71. https://doi.org/10.1007/s00429-010-0277-6.

Nunez, Paul L, Michael D Nunez, and Ramesh Srinivasan. 2019. "Multi-Scale Neural Sources of EEG : Genuine , Equivalent , and Representative . A Tutorial Review." Brain Topography 32 (2): 193-214. https://doi.org/10.1007/s10548-019-00701-3.

Nunez, Paul L, and Ramesh Srinivasan. 2006. Electric Fields of the Brain: The Neurophysics of EEG. Oxford University Press, USA.

Oosugi, Naoya, Keiichi Kitajo, Naomi Hasegawa, Yasuo Nagasaka, Kazuo Okanoya, and Naotaka Fujii. 2017. "A New Method for Quantifying the Performance of EEG Blind Source Separation Algorithms by Referencing a Simultaneously Recorded ECoG Signal." Neural Networks 93: 1-6.

Perge, János A., Shaomin Zhang, Wasim Q. Malik, Mark L. Homer, Sydney Cash, Gerhard Friehs, Emad N. Eskandar, John P. Donoghue, and Leigh R. Hochberg. 2014. "Reliability of Directional Information in Unsorted Spikes and Local Field Potentials Recorded in Human Motor Cortex." Journal of Neural Engineering 11 (4). https://doi.org/10.1088/1741- 
$2560 / 11 / 4 / 046007$.

Peron, Simon P., Jeremy Freeman, Vijay lyer, Caiying Guo, and Karel Svoboda. 2015. “A Cellular Resolution Map of Barrel Cortex Activity during Tactile Behavior." Neuron 86 (3): 783-99. https://doi.org/10.1016/j.neuron.2015.03.027.

Pesaran, Bijan, Martin Vinck, Gaute T. Einevoll, Anton Sirota, Pascal Fries, Markus Siegel, Wilson Truccolo, Charles E. Schroeder, and Ramesh Srinivasan. 2018. "Investigating Large-Scale Brain Dynamics Using Field Potential Recordings: Analysis and Interpretation." Nature Neuroscience 21 (7): 903-19. https://doi.org/10.1038/s41593-0180171-8.

Petersen, Carl C.H. 2019. "Sensorimotor Processing in the Rodent Barrel Cortex." Nature Reviews Neuroscience 20 (9): 533-46. https://doi.org/10.1038/s41583-019-0200-y.

Polley, Daniel B., Eugen Kvašňák, and Ron D. Frostig. 2004. "Naturalistic Experience Transforms Sensory Maps in the Adult Cortex of Caged Animals." Nature 429 (6987): 6771. https://doi.org/10.1038/nature02469.

Pothof, F., L. Bonini, M. Lanzilotto, A. Livi, L. Fogassi, G. A. Orban, O. Paul, and P. Ruther. 2016. "Chronic Neural Probe for Simultaneous Recording of Single-Unit, Multi-Unit, and Local Field Potential Activity from Multiple Brain Sites." Journal of Neural Engineering 13 (4): 1-13. https://doi.org/10.1088/1741-2560/13/4/046006.

Rollo, Patrick S., Matthew J. Rollo, Ping Zhu, Oscar Woolnough, and Nitin Tandon. 2021. "Oblique Trajectory Angles in Robotic Stereoelectroencephalography." Journal of Neurosurgery 135 (1): 245-54. https://doi.org/10.3171/2020.5.JNS20975.

Sacino, Matthew F., Sean S. Huang, John Schreiber, William D. Gaillard, and Chima O. Oluigbo. 2019. "Is the Use of Stereotactic Electroencephalography Safe and Effective in Children? A Meta-Analysis of the Use of Stereotactic Electroencephalography in Comparison to Subdural Grids for Invasive Epilepsy Monitoring in Pediatric Subjects." Clinical Neurosurgery. https://doi.org/10.1093/neuros/nyy466.

Schroeder, Charles E, Ashesh D Mehta, and Syndee J Givre. 1998. “A Spatiotemporal Profile of Visual System Activation Revealed by Current Source Density Analysis in the Awake Macaque." Cerebral Cortex (New York, NY: 1991) 8 (7): 575-92.

Seymour, John P, Nick B Langhals, David J Anderson, and Daryl R Kipke. 2011. "Novel Multi- 
Sided, Microelectrode Arrays for Implantable Neural Applications." Biomedical Microdevices 13 (3): 441-51.

Shannon, Claude Elwood. 1948. "A Mathematical Theory of Communication.” The Bell System Technical Journal 27 (3): 379-423.

Tadel, François, Sylvain Baillet, John C Mosher, Dimitrios Pantazis, and Richard M Leahy. 2011. "Brainstorm: A User-Friendly Application for MEG/EEG Analysis." Computational Intelligence and Neuroscience 2011.

Tandon, Nitin, BA Tong, ER Friedman, JA Johnson, G Von Allmen, MS Thomas, OA Hope, Giridhar P. Kalamangalam, Jeremy D. Slater, and Thompson SA. 2019. "Analysis of Morbidity and Outcomes Associated With Use of Subdural Grids vs Stereoelectroencephalography in Patients With Intractable Epilepsy." JAMA Neurol. Tandon, Nitin, Brian A Tong, Elliott R Friedman, Jessica A Johnson, Gretchen Von Allmen, Melissa S Thomas, Omotola A Hope, Giridhar P Kalamangalam, Jeremy D Slater, and Stephen A Thompson. 2019. "Analysis of Morbidity and Outcomes Associated with Use of Subdural Grids vs Stereoelectroencephalography in Patients with Intractable Epilepsy." JAMA Neurology 76 (6): 672-81.

Thomas, Tessy M, Robert W Nickl, Margaret C Thompson, Daniel N Candrea, Matthew S Fifer, David P McMullen, Luke E Osborn, Eric A Pohlmeyer, Manuel Anaya, and William S Anderson. 2020. "Simultaneous Classification of Bilateral Hand Gestures Using Bilateral Microelectrode Recordings in a Tetraplegic Patient." MedRxiv.

Toth, Marton, Kata Szilvia Papp, Noemi Gede, Kornelia Farkas, Sandor Kovacs, Jean Isnard, Koichi Hagiwara, et al. 2019. "Surgical Outcomes Related to Invasive EEG Monitoring with Subdural Grids or Depth Electrodes in Adults: A Systematic Review and Meta-Analysis." Seizure. https://doi.org/10.1016/j.seizure.2019.06.022.

Ulbert, István, Eric Halgren, Gary Heit, and George Karmos. 2001. "Multiple MicroelectrodeRecording System for Human Intracortical Applications." Journal of Neuroscience Methods 106 (1): 69-79. https://doi.org/10.1016/S0165-0270(01)00330-2.

Vakharia, Vejay N, Rachel Sparks, Aidan G O’Keeffe, Roman Rodionov, Anna Miserocchi, Andrew McEvoy, Sebastien Ourselin, and John Duncan. 2017. "Accuracy of Intracranial Electrode Placement for Stereoelectroencephalography: A Systematic Review and Metaanalysis." Epilepsia 58 (6): 921-32. 
Wellman, Steven M, James R Eles, Kip A Ludwig, John P Seymour, Nicholas J Michelson, William E McFadden, Alberto L Vazquez, and Takashi D Y Kozai. 2017. "A Materials Roadmap to Functional Neural Interface Design." Advanced Functional Materials.

Willett, Francis R, Donald T Avansino, Leigh R Hochberg, Jaimie M Henderson, and Krishna V Shenoy. n.d. "High-Performance Brain-to-Text Communication via Imagined Handwriting 1 2." https://doi.org/10.1101/2020.07.01.183384.

Yan, Han, Joel S. Katz, Melanie Anderson, Alireza Mansouri, Madison Remick, George M. Ibrahim, and Taylor J. Abel. 2019. "Method of Invasive Monitoring in Epilepsy Surgery and Seizure Freedom and Morbidity: A Systematic Review." Epilepsia.

https://doi.org/10.1111/epi.16315.

Yang, Meihua, Yuanshi Ma, Wei Li, Xianjun Shi, Zhi Hou, Ning An, Chunqing Zhang, Lihong Liu, Hui Yang, and Dong Zhang. 2017. "A Retrospective Analysis of Stereoelectroencephalography and Subdural Electroencephalography for Preoperative Evaluation of Intractable Epilepsy." Stereotactic and Functional Neurosurgery 95 (1): 1320. 